\title{
Limit theorems for weakly subcritical branching processes in random environment*
}

\author{
V.I. Afanasyev ${ }^{\dagger}$ C. Böinghoff ${ }^{\ddagger}$ \\ G. Kersting ${ }^{\S}$ V.A. VAtutin $₫$
}

May 29, 2022

\begin{abstract}
For a branching process in random environment it is assumed that the offspring distribution of the individuals varies in a random fashion, independently from one generation to the other. Interestingly there is the possibility that the process may at the same time be subcritical and, conditioned on nonextinction, 'supercritical'. This so-called weakly subcritical case is considered in this paper. We study the asymptotic survival probability and the size of the population conditioned on non-extinction. Also a functional limit theorem is proven, which makes the conditional supercriticality manifest. A main tool is a new type of functional limit theorem for conditional random walks.
\end{abstract}

MSC 2000 subject classifications. Primary 60J80, Secondary 60G50, 60F17

Key words and phrases. Branching process, random environment, random walk, change of measure, survival probability, functional limit theorem

\footnotetext{
${ }^{*}$ This paper is part of a project supported by the German Research Foundation (DFG) and the Russian Foundation of Basic Research (Grant DFG-RFBR 08-01-91954)

${ }^{\dagger}$ Department of Discrete Mathematics, Steklov Mathematical Institute, 8 Gubkin Street, 119991 Moscow, Russia, viafan@mail.ru

¥Fachbereich Mathematik, Universität Frankfurt, Fach 187, D-60054 Frankfurt am Main, Germany, boeinghoff@math.uni-frankfurt.de

${ }^{\S}$ Fachbereich Mathematik, Universität Frankfurt, Fach 187, D-60054 Frankfurt am Main, Germany, kersting@math.uni-frankfurt.de

IDepartment of Discrete Mathematics, Steklov Mathematical Institute, 8 Gubkin Street, 119991 Moscow, Russia, vatutin@mi.ras.ru
} 


\section{Introduction and main results}

For a branching process in random environment it is assumed that the offspring distribution of the individuals varies in a random fashion, independently from one generation to the other. Conditioned on the environment individuals reproduce independently of each other. Let $Q_{n}$ be the random offspring distribution of an individual at generation $n-1$ and let $Z_{n}$ denote the number of individuals at generation $n$. Then $Z_{n}$ is the sum of $Z_{n-1}$ independent random variables, each of which has distribution $Q_{n}$. To give a formal definition let $\Delta$ be the space of probability measures on $\mathbb{N}_{0}:=\{0,1, \ldots\}$, which equipped with the metric of total variation is a Polish space. Let $Q$ be a random variable taking values in $\Delta$. Then, an infinite sequence $\Pi=\left(Q_{1}, Q_{2}, \ldots\right)$ of i.i.d. copies of $Q$ is said to form a random environment. A sequence of $\mathbb{N}_{0}$-valued random variables $Z_{0}, Z_{1}, \ldots$ is called a branching process in the random environment $\Pi$, if $Z_{0}$ is independent of $\Pi$ and given $\Pi$ the process $Z=\left(Z_{0}, Z_{1}, \ldots\right)$ is a Markov chain with

$$
\mathcal{L}\left(Z_{n} \mid Z_{n-1}=z, \Pi=\left(q_{1}, q_{2}, \ldots\right)\right)=q_{n}^{* z}
$$

for every $n \in \mathbb{N}, z \in \mathbb{N}_{0}$ and $q_{1}, q_{2}, \ldots \in \Delta$, where $q^{* z}$ is the $z$-fold convolution of the measure $q$. The corresponding probability measure on the underlying probability space will be denoted by $\mathbb{P}$. In the following we assume that the process starts with a single founding ancestor, $Z_{0}=1$ a.s., and (without loss of generality) that $\mathbb{P}\{Q(\{0\})=1\}=0$. Note that in general $Z$ is not the superposition of $Z_{0}$ independent copies of the process started at $Z_{0}=1$.

It turns out that the asymptotic behavior of the generation size process $Z$ is determined in the main by the associated random walk $S=\left(S_{n}\right)_{n \geq 0}$. This random walk has initial state $S_{0}=0$ and increments $X_{n}=S_{n}-S_{n-1}, n \geq 1$ defined as

$$
X_{n}:=\log m\left(Q_{n}\right),
$$

where

$$
m(q):=\sum_{y=0}^{\infty} y q(\{y\})
$$

is the mean of the offspring distribution $q \in \Delta$. In view of (1.1) and the assumption $Z_{0}=1$ a.s. the conditional expectation of $Z_{n}$ given the environment $\Pi$ can be expressed by means of $(S)_{n \in \mathbb{N}_{0}}$ as

$$
\mathbb{E}\left[Z_{n} \mid \Pi\right]=\prod_{k=1}^{n} m\left(Q_{k}\right)=\exp \left(S_{n}\right) \quad \mathbb{P} \text {-a.s. }
$$


Averaging over the environment gives

$$
\mathbb{E}\left[Z_{n}\right]=(\mathbb{E}[m(Q)])^{n} .
$$

If the random walk $(S)_{n \in \mathbb{N}_{0}}$ drifts to $-\infty$, then the branching process is said to be subcritical. In case $X=\log m(Q)$ has finite mean, subcriticality corresponds to $\mathbb{E}[X]<0$. For such processes the conditional non-extinction probability at $n$ decays at an exponential rate for almost every environment. This fact is an immediate consequence of the strong law of large numbers and the first moment estimate

$$
\begin{aligned}
& \mathbb{P}\left\{Z_{n}>0 \mid \Pi\right\}=\min _{0 \leq k \leq n} \mathbb{P}\left\{Z_{k}>0 \mid \Pi\right\} \\
& \quad \leq \min _{0 \leq k \leq n} \mathbb{E}\left[Z_{k} \mid \Pi\right]=\exp \left(\min _{0 \leq k \leq n} S_{k}\right) \quad \mathbb{P} \text {-a.s. }
\end{aligned}
$$

As was first observed by Afanasyev [1] and later independently by Dekking [14] there are three possibilities for the asymptotic behavior of subcritical branching processes. They are called the weakly subcritical, the intermediate subcritical and the strongly subcritical case. Here we study the weakly subcritical case.

The present article is part of several publications having started with [4, [5], in which we try to develop characteristic properties of the different cases. For a comparative discussion we refer the reader to [11. One purpose of this paper is to make the methods, developed in [4] for criticality, also available for weak subcriticality.

Assumption A1. The process $Z$ is weakly subcritical, that is, there is a number $0<\beta<1$ such that

$$
\mathbb{E}\left[X e^{\beta X}\right]=0
$$

This implies $-\infty \leq \mathbb{E}[X]<0$, thus $(S)_{n \in \mathbb{N}_{0}}$ has negative drift with respect to $\mathbb{P}$. Assumption $\mathrm{A} 1$ is somewhat weaker than $\mathbb{E}[X]<0<\mathbb{E}\left[X e^{X}\right]<\infty$, which is a customary condition for weak subcriticality. The assumption suggests to change to the measure $\mathbf{P}$ with expectation $\mathbf{E}$. For any $n \in \mathbb{N}$ and any measurable, bounded function $\varphi: \Delta^{n} \times \mathbb{N}_{0}^{n+1} \rightarrow \mathbb{R}$, the measure $\mathbf{P}$ is given by $\mathbf{E}\left[\varphi\left(Q_{1}, \ldots, Q_{n}, Z_{0}, \ldots, Z_{n}\right)\right]:=\gamma^{-n} \mathbb{E}\left[\varphi\left(Q_{1}, \ldots, Q_{n}, Z_{0}, \ldots, Z_{n}\right) e^{\beta\left(S_{n}-S_{0}\right)}\right]$, 
with

$$
\gamma:=\mathbb{E}\left[e^{\beta X}\right] .
$$

(We include $S_{0}$ in the above expression, because later we shall also consider cases where $S_{0} \neq 0$.) Then $\mathbb{E}\left[X e^{\beta X}\right]=0$ translates into

$$
\mathbf{E}[X]=0 .
$$

Thus $(S)_{n \in \mathbb{N}_{0}}$ becomes a recurrent random walk under $\mathbf{P}$.

As to the regularity of the distribution of $X$ we make the following assumptions.

Assumption A2. The distribution of $X$ has finite variance with respect to $\mathbf{P}$ or (more generally) belongs to the domain of attraction of some stable law with index $\alpha \in(1,2]$. It is non-lattice.

Since $\mathbf{E}[X]=0$ this means that there is an increasing sequence of positive numbers

$$
a_{n}=n^{1 / \alpha} \ell_{n}
$$

with a slowly varying sequence $\ell_{1}, \ell_{2}, \ldots$ such that for $n \rightarrow \infty$

$$
\mathbf{P}\left\{S_{n} / a_{n} \in d x\right\} \rightarrow s(x) d x
$$

weakly, where $s(x)$ denotes the density of the limiting stable law. Below the local version of this statement will mainly be used. Note that due to the change of measure $X^{-}$always has finite variance and an infinite variance may only arise from $X^{+}$. Then the stable law $s(x) d x$ is completely skewed towards the positive real axis. Nevertheless it is not a one sided law: $s(x)$ is strictly positive everywhere, as this is the case for stable laws with finite expectation.

Remark. In [4 we studied branching processes in a critical random environment under the assumption that the random walk $(S)_{n \in \mathbb{N}_{0}}$ fulfils Spitzer's condition. In general this condition is less restrictive than A2. However, if $X^{-}$has finite second moment, then A2 is equivalent to Spitzer's condition (cf. [15]). 
Our last assumption on the environment concerns the standardized truncated second moment of $Q$,

$$
\zeta(a):=\sum_{y=a}^{\infty} y^{2} Q(\{y\}) / m(Q)^{2}, \quad a \in \mathbb{N} .
$$

Assumption A3. For some $\varepsilon>0$ and some $a \in \mathbb{N}$

$$
\mathbf{E}\left[\left(\log ^{+} \zeta(a)\right)^{\alpha+\varepsilon}\right]<\infty,
$$

where $\log ^{+} x:=\log (\max (x, 1))$.

Remark For examples where this assumption is fulfilled, see [4]. In particular our results hold for binary branching processes in random environment (where individuals have either two children or none) and for cases where $Q$ is a.s. a Poisson distribution or a.s. a geometric distribution.

We now come to the main results of the paper. All our limit theorems are under the law $\mathbb{P}$ which is what is called the annealed approach. The first theorem describes the asymptotic behaviour of the non-extinction probability at generation $n$. In the following, for sequences $\left(d_{n}\right)$ and $\left(m_{n}\right)$, we write $d_{n} \sim m_{n}$ if $d_{n} / m_{n} \rightarrow 1$ as $n \rightarrow \infty$.

Theorem 1.1. Assume A1 to A3. Then there exists a number $0<\kappa<\infty$ such that

$$
\mathbb{P}\left\{Z_{n}>0\right\} \sim \kappa \mathbb{P}\left\{\min \left(S_{1}, \ldots, S_{n}\right) \geq 0\right\} \quad \text { as } n \rightarrow \infty .
$$

We point out that the same result holds in the critical case (see [4), whereas it is no longer true in the moderate and strongly subcritical case (see e.g. [19]). As a corollary we obtain from Proposition 2.1 below the following result.

Corollary 1.2. Under A1 to A3 there is a number $0<\kappa^{\prime}<\infty$ such that

$$
\mathbb{P}\left\{Z_{n}>0\right\} \sim \kappa^{\prime} \frac{\gamma^{n}}{n a_{n}} .
$$

The next theorem gives convergence of the laws of $Z_{n}$, conditioned on survival.

Theorem 1.3. Under $A 1$ to $A 3$ the conditional laws $\mathcal{L}\left(Z_{n} \mid Z_{n}>0\right), n \geq 1$, converge weakly to some probability distribution on the natural numbers. Moreover the sequence $\mathbb{E}\left[Z_{n}^{\vartheta} \mid Z_{n}>0\right]$ is bounded for any $\vartheta<\beta$, implying convergence to the corresponding moment of the limit distribution. 
Our last theorem describes the limiting behavior of the rescaled generation size process $e^{-S_{k}} Z_{k}$ for $r_{n} \leq k \leq n-r_{n}$, where $\left(r_{n}\right)$ is a sequence of natural numbers with $r_{n} \rightarrow \infty$ (and certainly $r_{n}<n / 2$ ). Thus we consider the process $Y^{n}=\left\{Y_{t}^{n}, t \in[0,1]\right\}$, given by

$$
Y_{t}^{n}:=\exp \left(-S_{r_{n}+\left\lfloor\left(n-2 r_{n}\right) t\right\rfloor}\right) Z_{r_{n}+\left\lfloor\left(n-2 r_{n}\right) t\right\rfloor} .
$$

This process has asymptotically paths of a constant random value. More precisely:

Theorem 1.4. Under $A 1$ to $A 3$, there is a process $\left\{W_{t}, t \in[0,1]\right\}$ such that as $n \rightarrow \infty$

$$
\mathcal{L}\left(Y_{t}^{n}, t \in[0,1] \mid Z_{n}>0\right) \Rightarrow \mathcal{L}\left(W_{t}, t \in[0,1]\right)
$$

weakly in the Skorohod space $D[0,1]$. Moreover, there is a random variable $W$ such that $W_{t}=W$ a.s. for all $t \in[0,1]$ and

$$
\mathbb{P}\{0<W<\infty\}=1
$$

Weaker versions of this results can be found in [3, 19.

Thus we have the following scenario in the weakly subcritical case (being different from other cases): Given $Z_{n}>0$ the value of $Z_{k}$ is of bounded order for $k$ close to 0 and close to $n$. Inbetween $S_{k}$ takes large values, as can be seen from the proofs. In the first part, roughly up to time $\lfloor\epsilon n\rfloor, S_{k}$ is increasing exponentially fast, and the growth of $Z_{k}$ resembles that of supercritical growth. Then $Z_{k}$ follows the value of $e^{S_{k}}=\mathbb{E}\left[Z_{k} \mid \Pi\right]$ in a completely deterministic manner, up to a random factor $W>0$. Afterwards this behaviour persists as long as $S_{k}$ remains large. Only at the end $S_{k}$ returns to 0 in the manner of a random walk excursion (as in [21]), and $Z_{k}$ is no longer tied to $S_{k}$. For further explanations we refer to [11].

For the proof we develop several limit theorems for random walks $\left(S_{n}\right)_{n \in \mathbb{N}_{0}}$, conditioned to stay positive up to time $n$, for functionals, which depend primarily on the values of $S_{k}$ with $k$ being close to 0 or to $n$. These theorems are presented in the following section. The proofs of the theorems are given in the closing section. 


\section{Some limit theorems for random walks}

In this section, we develop conditional limit theorems for a class of oscillating random walks without refering to branching processes.

Let $X_{1}, X_{2}, \ldots$ be a sequence of i.i.d. real-valued random variables and $S_{0}$ independent of $X_{1}, X_{2}, \ldots$. The random walk $S=\left(S_{n}\right)_{n \in \mathbb{N}_{0}}$ is defined by

$$
S_{n}=S_{0}+X_{1}+\cdots+X_{n}
$$

Our results are valid under a more general condition than A2, namely:

Assumption B. There are numbers $a_{n}>0$ such that $S_{n} / a_{n}$ converges in distribution to a law which is neither concentrated on $\mathbb{R}^{+}$nor on $\mathbb{R}^{-}$.

As is well-known the limit distribution is strictly stable with index $\alpha \in(0,2]$ with a density $s(x)$, such that $s(0)>0$.

Our theorems rely on conclusions from the theory of random walks, which we put together in this section. They rest on and substantially extend results due to Afanasyev [2], Bertoin and Doney [9], Hirano [20], Iglehart [21, Keener 22], and others.

In the sequel we shall also consider the possibility that the random walk starts from any point $x \in \mathbb{R}$ or from an initial distribution $\mu$. In such cases we write for probabilities as usual $\mathbf{P}_{x}\{\cdot\}$ or $\mathbf{P}_{\mu}\{\cdot\}$. We write $\mathbf{P}$ instead of $\mathbf{P}_{0}$.

Duality will be an important tool later. Recall that given $n$ one may consider the dual objects $Q_{i}^{\prime}:=Q_{n-i+1}, X_{i}^{\prime}:=X_{n-i+1}$ for $i=1, \ldots, n$. Then the dual random walk is given by $S_{i}^{\prime}:=X_{1}^{\prime}+\cdots+X_{i}^{\prime}=S_{n}-S_{n-i}, S_{0}^{\prime}=0$. We refrain from indicating the dependence on $n$ in the notation.

Let us introduce

$$
M_{n}:=\max \left(S_{1}, \ldots, S_{n}\right), \quad L_{n}:=\min \left(S_{1}, \ldots, S_{n}\right)
$$

and the right-continuous functions $u: \mathbb{R} \rightarrow \mathbb{R}$ and $v: \mathbb{R} \rightarrow \mathbb{R}$ given by

$$
\begin{array}{ll}
u(x):=1+\sum_{k=1}^{\infty} \mathbf{P}\left\{-S_{k} \leq x, M_{k}<0\right\}, & x \geq 0, \\
v(x):=1+\sum_{k=1}^{\infty} \mathbf{P}\left\{-S_{k}>x, L_{k} \geq 0\right\}, & x \leq 0
\end{array}
$$


and 0 elsewhere. In particular $u(0)=v(0)=1$. It is well-known that $u(x)=$ $O(x), v(-x)=O(x)$ for $x \rightarrow \infty$.

2.1 Large deviations for random walks. The following precise large deviation estimates are extensions of known results. Some related results can be found in [16]. Recall that $s(x)$ denotes the limiting density of $S_{n} / a_{n}$ and that $s(0)>0$ under Assumption B.

Proposition 2.1. For $\theta>0, x \geq 0$,

$$
\mathbf{E}_{x}\left[e^{-\theta S_{n}} ; L_{n} \geq 0\right] \sim s(0) b_{n} u(x) \int_{0}^{\infty} e^{-\theta z} v(-z) d z,
$$

and for $\theta>0, x \leq 0$

$$
\mathbf{E}_{x}\left[e^{\theta S_{n}} ; M_{n}<0\right] \sim s(0) b_{n} v(x) \int_{0}^{\infty} e^{-\theta z} u(z) d z
$$

with

$$
b_{n}=\left(a_{n} n\right)^{-1} .
$$

For the proof we need the following lemma.

Lemma 2.2. Let $\left(\beta_{n}\right)$ be a regularly varying sequence with $\sum_{k=0}^{\infty} \beta_{k}<\infty$ and $d, e>0$.

i) If $\delta_{n} \sim d \beta_{n}, \eta_{n} \sim e \beta_{n}$, then $\sum_{i=0}^{n} \delta_{i} \eta_{n-i} \sim c \beta_{n}$ with $c:=d \sum_{k=0}^{\infty} \eta_{k}+$ $e \sum_{k=0}^{\infty} \delta_{k}$ as $n \rightarrow \infty$.

ii) If $\sum_{k=0}^{\infty} \alpha_{k} t^{k}=\exp \left(\sum_{k=0}^{\infty} \beta_{k} t^{k}\right)$ for $|t|<1$, then $\alpha_{n} \sim c \beta_{n}$ with $c:=$ $\sum_{k=0}^{\infty} \alpha_{k}$ as $n \rightarrow \infty$.

Proof. i) is a well-known elementary fact and ii) is a special case of Theorem 1 in [13].

Proof of Proposition 2.1. Both claims are proven along the same lines. Since the first one has been considered (under stronger conditions) by Hirano [20, let us turn to the second statement. By Assumption B and Stone's Local Limit Theorem (cf. [10, section 8.4.1) for any interval $I$ of length $|I|$

$$
a_{n} \mathbf{P}\left\{S_{n} \in I\right\} \rightarrow s(0)|I| .
$$


Moreover the local limit theorem implies that there is a $c>0$ such that

$$
\mathbf{P}\left\{S_{n} \in I\right\} \leq c / a_{n}
$$

uniformly in $n$ and all intervals $I$ of length at most 1 . Therefore for $\theta>0$

$$
\sup _{n} a_{n} \mathbf{E}\left[e^{\theta S_{n}} ; S_{n}<0\right] \leq \sup _{n} \sum_{k=0}^{\infty} a_{n} \mathbf{P}\left\{-k-1 \leq S_{n}<-k\right\} e^{-\theta k}<\infty
$$

Also for any $h>0$,

$$
\begin{gathered}
a_{n} \sum_{k=0}^{\infty} e^{-\theta(k+1) h} \mathbf{P}\left\{-(k+1) h \leq S_{n}<-k h\right\} \leq a_{n} \mathbf{E}\left[e^{\theta S_{n}} ; S_{n}<0\right] \\
\leq a_{n} \sum_{k=0}^{\infty} e^{-\theta k h} \mathbf{P}\left\{-(k+1) h \leq S_{n}<-k h\right\}
\end{gathered}
$$

Now taking the limit $n \rightarrow \infty$, the limit and the sums interchange due to (2.2) and dominated convergence. Then taking the limit $h \rightarrow 0$ yields

$$
a_{n} \mathbf{E}\left[e^{\theta S_{n}} ; S_{n}<0\right] \rightarrow s(0) \int_{0}^{\infty} e^{-\theta z} d z=\frac{s(0)}{\theta} .
$$

Next the Baxter identity says that, for $|t|<1$ and $\theta>0$

$$
1+\sum_{k=1}^{\infty} t^{k} \mathbf{E}\left[e^{\theta S_{k}} ; M_{k}<0\right]=\exp \left(\sum_{k=1}^{\infty} \frac{t^{k}}{k} \mathbf{E}\left[e^{\theta S_{k}} ; S_{k}<0\right]\right)
$$

(cf. [18, chapter XVIII.3 or [10], chapter 8.9). Also $\sum_{k=1}^{\infty} k^{-1} \mathbf{E}\left[e^{\theta S_{k}} ; S_{k}<\right.$ $0]<\infty$. Thus from Lemma 2.2 ii) it follows

$$
\begin{aligned}
n a_{n} \mathbf{E}\left[e^{\theta S_{n}} ; M_{n}<0\right] & \rightarrow \frac{s(0)}{\theta}\left(1+\sum_{k=1}^{\infty} \mathbf{E}\left[e^{\theta S_{k}} ; M_{k}<0\right]\right) \\
& =s(0) \int_{0}^{\infty} e^{-\theta z} u(z) d z .
\end{aligned}
$$

In much the same way Hirano [20] obtained

$$
n a_{n} \mathbf{E}\left[e^{-\theta S_{n}} ; L_{n} \geq 0\right] \rightarrow s(0) \int_{0}^{\infty} e^{-\theta z} v(-z) d z .
$$

by means of the corresponding Baxter identity $\left(L_{n} \geq 0\right.$ and $S_{n} \geq 0$ replacing $M_{n}<0$ and $\left.S_{n}<0\right)$. By the continuity theorem for Laplace transforms this generalizes to

$$
\begin{gathered}
n a_{n} \mathbf{E}\left[e^{\theta S_{n}} ; M_{n}<0, S_{n}>-x\right] \rightarrow s(0) \int_{0}^{x} e^{-\theta z} u(z) d z \\
n a_{n} \mathbf{E}\left[e^{-\theta S_{n}} ; L_{n} \geq 0, S_{n}<x\right] \rightarrow s(0) \int_{0}^{x} e^{-\theta z} v(-z) d z,
\end{gathered}
$$


which for finite $x \geq 0$ now holds for every $\theta \in \mathbb{R}$. Note that the limit measures involved here have densities with respect to the Lebesgue measure and thus have no point masses, so that the convergence holds for any finite $x \geq 0$.

Next let $x<0$. By means of duality

$$
\begin{aligned}
\mathbf{E}\left[e^{\theta S_{n}} ; M_{n}<-x\right]= & \sum_{i=0}^{n-1} \mathbf{E}\left[e^{\theta S_{n}} ; S_{0}, \ldots, S_{i} \leq S_{i}<-x, S_{i}>S_{i+1}, \ldots, S_{n}\right] \\
& +\mathbf{E}\left[e^{\theta S_{n}} ; S_{0}, \ldots, S_{n} \leq S_{n}<-x\right] \\
= & \sum_{i=0}^{n} \mathbf{E}\left[e^{\theta S_{i}} ; L_{i} \geq 0, S_{i}<-x\right] \cdot \mathbf{E}\left[e^{\theta S_{n-i}} ; M_{n-i}<0\right] .
\end{aligned}
$$

This formula together with (2.3), (2.5) and the equations (note that $v(-z)$ is left continuous for $z>0$ and that $v(0)=v(0-)=1$ )

$$
\begin{aligned}
& 1+\sum_{k=1}^{\infty} \mathbf{E}\left[e^{\theta S_{k}} ; L_{k} \geq 0, S_{k}<-x\right] \\
& =1+\int_{(0,-x)} e^{\theta z} d v(-z)=e^{-\theta x} v(x)-\theta \int_{0}^{-x} e^{\theta z} v(-z) d z, \\
& 1+\sum_{k=1}^{\infty} \mathbf{E}\left[e^{\theta S_{k}} ; M_{k}<0\right]=\theta \int_{0}^{\infty} e^{-\theta z} u(z) d z
\end{aligned}
$$

imply by means of Lemma 2.2 i) for $\theta>0$ and $x<0$

$$
n a_{n} \mathbf{E}\left[e^{\theta S_{n}} ; M_{n}<-x\right] \rightarrow s(0) e^{-\theta x} v(x) \int_{0}^{\infty} e^{-\theta z} u(z) d z,
$$

which is equivalent to our claim.

Related to these results are the following upper estimates. Their proofs shed some light on how the term $b_{n}=\left(a_{n} n\right)^{-1}$ comes into play.

Proposition 2.3. There is a number $c>0$ such that uniformly for all $x, y \geq 0$ and all $n$

$$
\mathbf{P}_{x}\left\{L_{n} \geq 0, y-1 \leq S_{n}<y\right\} \leq c b_{n} u(x) v(-y),
$$

whereas for $x, y \leq 0$

$$
\mathbf{P}_{x}\left\{M_{n}<0, y \leq S_{n}<y+1\right\} \leq c b_{n} v(x) u(-y) .
$$


Proof. As before, we prove the latter statement. Let $S^{\prime}$ be the dual random walk and $L_{i}^{\prime}, i=1, \ldots, n$, the corresponding minima. Denote

$$
\begin{aligned}
A_{n} & :=\left\{M_{\lfloor n / 3\rfloor}<-x\right\} \\
A_{n}^{\prime} & :=\left\{L_{\lfloor n / 3\rfloor}^{\prime} \geq y\right\}, \\
A_{n}^{\prime \prime} & :=\left\{y-x \leq S_{n}<y-x+1\right\} \\
& =\left\{y-x-T_{n} \leq S_{\lfloor 2 n / 3\rfloor}-S_{\lfloor n / 3\rfloor}<y-x-T_{n}+1\right\},
\end{aligned}
$$

with $T_{n}:=S_{\lfloor n / 3\rfloor}+S_{n}-S_{\lfloor 2 n / 3\rfloor}$. Let $\mathcal{A}_{n}$ be the $\sigma$-field generated by $X_{1}, \ldots, X_{\lfloor n / 3\rfloor}$ and $X_{\lfloor 2 n / 3\rfloor+1}, \ldots, X_{n}$. Then $T_{n}$ is $\mathcal{A}_{n}$-measurable, whereas $S_{\lfloor 2 n / 3\rfloor}-S_{\lfloor n / 3\rfloor}$ is independent of $\mathcal{A}_{n}$, consequently from (2.1) and the fact that $\left(a_{n}\right)$ is regularly varying there is a $c>0$ such that

$$
\mathbf{P}\left\{A_{n}^{\prime \prime} \mid \mathcal{A}_{n}\right\} \leq c a_{n}^{-1} .
$$

Since $A_{n}, A_{n}^{\prime}$ are $\mathcal{A}_{n}$-measurable and independent, it follows

$$
\mathbf{P}\left\{A_{n} \cap A_{n}^{\prime} \cap A_{n}^{\prime \prime}\right\} \leq c a_{n}^{-1} \mathbf{P}\left\{A_{n}\right\} \mathbf{P}\left\{A_{n}^{\prime}\right\} .
$$

Moreover from Lemma 2.1 in [4] there is a number $\rho \in(0,1)$ and a slowly varying sequence $l_{1}, l_{2}, \ldots$ such that

$$
\mathbf{P}\left\{L_{n} \geq y\right\} \leq c_{1} u(-y) n^{-\rho} l_{n}, \quad \mathbf{P}\left\{M_{n}<-x\right\} \leq c_{2} v(x) n^{\rho-1} l_{n}^{-1},
$$

and we end up with the uniform estimate

$$
\mathbf{P}\left\{A_{n} \cap A_{n}^{\prime} \cap A_{n}^{\prime \prime}\right\} \leq c v(x) u(-y) b_{n}
$$

for $c$ sufficiently large. Now since $M_{\lfloor n / 3\rfloor} \leq M_{n}$ and $L_{\lfloor n / 3\rfloor}^{\prime} \leq S_{n}-M_{n}$,

$$
\left\{M_{n}<-x, y-x \leq S_{n}<y-x+1\right\} \subset A_{n} \cap A_{n}^{\prime} \cap A_{n}^{\prime \prime},
$$

and the claim follows.

Corollary 2.4. For any $\theta>0$ there is a $c>0$ (depending on $\theta$ ) such that for all $x, y \geq 0$

$$
\mathbf{E}_{x}\left[e^{-\theta S_{n}} ; L_{n} \geq 0, S_{n} \geq y\right] \leq c b_{n} u(x) v(-y) e^{-\theta y}
$$

and for all $x, y \leq 0$

$$
\mathbf{E}_{x}\left[e^{\theta S_{n}} ; M_{n}<0, S_{n}<y\right] \leq c b_{n} u(-y) v(x) e^{\theta y} .
$$


Proof. Again we consider the latter statement. Let $\theta>0$ and $x, y \leq 0$. We use the inequalities

$$
\begin{aligned}
& u(x+y) \leq u(x)+u(y), \\
& u(x+y) \leq 2 u(x) u(y) .
\end{aligned}
$$

The first inequality is a consequence of the representation of $u$ as renewal function $(u(x)$ is the expected number of ladder points in the interval $[0,-x]$ plus one; see [18], chapter XII). The second inequality follows directly from the first one (as $u(x) \geq 1$ for all $x \geq 0)$. Then

$$
\begin{aligned}
\mathbf{E}_{x}\left[e^{\theta S_{n}} ;\right. & \left.M_{n}<0, S_{n}<y\right] \\
& \leq \sum_{k=1}^{\infty} e^{\theta(y-k)} \mathbf{P}_{x}\left\{M_{n}<0,-k+y \leq S_{n}<-k+y+1\right\} \\
& \leq e^{\theta y} \sum_{k=1}^{\infty} e^{-\theta k} c b_{n} v(x) u(k-y-1) \\
& \leq 2 c b_{n} e^{\theta y} v(x) u(-y) \sum_{k=0}^{\infty} e^{-\theta(k+1)} u(k) .
\end{aligned}
$$

2.2 The probability measures $\mathbf{P}^{+}$and $\mathbf{P}^{-}$. The fundamental properties of $u, v$ are the identities

$$
\begin{aligned}
& \mathbf{E}[u(x+X) ; X+x \geq 0]=u(x), \quad x \geq 0, \\
& \mathbf{E}[v(x+X) ; X+x<0]=v(x), \quad x \leq 0,
\end{aligned}
$$

which hold for any oscillating random walk.

We use them to introduce the probability measures $\mathbf{P}^{+}$and $\mathbf{P}^{-}$. The construction procedure is standard and explained for $\mathbf{P}^{+}$in detail in [4] and 9].

The probability measures $\mathbf{P}_{x}^{+}, x \geq 0$ are defined as follows. Assume that the random walk $\left(S_{n}\right)_{n \in \mathbb{N}_{0}}$ is adapted to some filtration $\mathcal{F}=\left(\mathcal{F}_{n}\right)$ and that $X_{n+1}$ is independent ot $\mathcal{F}_{n}$ for all $n \geq 0$. For every sequence $R_{0}, R_{1}, \ldots$ of $\mathcal{S}$-valued random variables, adapted to $\mathcal{F}$ and every integrable function $g: \mathcal{S}^{n+1} \rightarrow \mathbb{R}$, $n \in \mathbb{N}, \mathbf{P}_{x}^{+}$fulfills

$$
\mathbf{E}_{x}^{+}\left[g\left(R_{0}, \ldots, R_{n}\right)\right]=\frac{1}{u(x)} \mathbf{E}_{x}\left[g\left(R_{0}, \ldots, R_{n}\right) u\left(S_{n}\right) ; L_{n} \geq 0\right], n \in \mathbb{N}_{0} .
$$


This is the Doob transform from the theory of Markov chains. In particular, under $\mathbf{P}^{+} S_{0}, S_{1}, \ldots$ is a Markov process with state space $[0, \infty)$ and transition probabilities

$$
P^{+}(x, d y):=\frac{1}{u(x)} \mathbf{P}\{x+X \in d y\} u(y) 1_{\{y \geq 0\}}, \quad x \geq 0 .
$$

It is the random walk conditioned never to enter $(-\infty, 0)$.

Similarly $v$ gives rise to probability measures $\mathbf{P}_{x}^{-}, x \leq 0$, characterized by the equation

$$
\mathbf{E}_{x}^{-}\left[g\left(R_{0}, \ldots, R_{n}\right)\right]=\frac{1}{v(x)} \mathbf{E}_{x}\left[g\left(R_{0}, \ldots, R_{n}\right) v\left(S_{n}\right) ; M_{n}<0\right] .
$$

Under $\mathbf{P}_{x}^{-}$the process $S_{0}, S_{1}, \ldots$ becomes a Markov chain with state space $\mathbb{R}^{-}$ and transition kernel

$$
P^{-}(x, d y):=\frac{1}{v(x)} \mathbf{P}\{x+X \in d y\} v(y) 1_{\{y<0\}}, \quad x \leq 0 .
$$

Note that $P^{-}(x,[0, \infty))=0$, thus the Markov process never enters $[0, \infty)$ again. It may, however, start from the boundary $x=0$. Intuitively it is the random walk conditioned never to return to $[0, \infty)$.

Remark. Under $\mathbf{P}_{x}^{+}$the process $(S)_{n \in \mathbb{N}_{0}}$ may return to 0, however, under $\mathbf{P}_{x}^{-}$this possibility is excluded. We remark that this subtlety has little impact: For $x<0$ there is a difference only for those $x$, where $v(x) \neq v(x-)$, that is for at most countably many $x$. In particular no difference occurs, if one considers (as in the sequel) measures $\mathbf{P}_{\nu}^{-}$having an initial distribution $\nu$ without atoms.

2.3 Some conditional limit theorems. By means of the measures $\mathbf{P}_{x}^{+}, \mathbf{P}_{x}^{-}$ we now generalize a result due to Hirano [20] on the limit behavior of certain conditional distributions. For $\theta>0$, let $\mu_{\theta}, \nu_{\theta}$ be the probability measures on $\mathbb{R}^{+}$and $\mathbb{R}^{-}$given by their densities

$$
\mu_{\theta}(d z):=c_{1} e^{-\theta z} u(z) 1_{\{z \geq 0\}} d z, \quad \nu_{\theta}(d z):=c_{2} e^{\theta z} v(z) 1_{\{z<0\}} d z
$$

with $c_{1}^{-1}=c_{1 \theta}^{-1}=\int_{0}^{\infty} e^{-\theta z} u(z) d z, c_{2}^{-1}=c_{2 \theta}^{-1}=\int_{-\infty}^{0} e^{\theta z} v(z) d z$.

As above let $R_{0}, R_{1}, \ldots$ be a sequence of $\mathcal{S}$-valued random variables, adapted to $\mathcal{F}$. Also let $Q_{1}, Q_{2}, \ldots$ be a sequence of i.i.d. random variables with values 
in some space $\mathcal{D}$ and adapted to $\mathcal{F}$, such that $Q_{n+1}$ is independent of $\mathcal{F}_{n}$ for all $n \geq 0$. Additionally, assume that $X_{i}$ is $\sigma\left(Q_{i}\right)$-measurable for all $i \geq 1$.

Proposition 2.5. For given $\theta>0$ and $i, j \geq 0$ let $U:=g\left(R_{0}, \ldots, R_{i}\right)$ and $V:=$ $h\left(Q_{1}, \ldots, Q_{j}\right)$ be real-valued, bounded random variables with suitable bounded, measurable functions $g: \mathcal{S}^{i+1} \rightarrow \mathbb{R}, h: \mathcal{D}^{j} \rightarrow \mathbb{R}$. Also let $\varphi: \mathbb{R} \rightarrow \mathbb{R}$ be bounded and continuous. Denote $\tilde{V}_{n}:=h\left(Q_{n}, \ldots, Q_{n-j+1}\right)$. Then for $x \geq 0$

$$
\frac{\mathbf{E}_{x}\left[U \tilde{V}_{n} \varphi\left(S_{n}\right) e^{-\theta S_{n}} ; L_{n} \geq 0\right]}{\mathbf{E}_{x}\left[e^{-\theta S_{n}} ; L_{n} \geq 0\right]} \rightarrow \mathbf{E}_{x}^{+}[U] \mathbf{E}_{\nu_{\theta}}^{-}\left[V \varphi\left(-S_{0}\right)\right]
$$

and for $x \leq 0$

$$
\frac{\mathbf{E}_{x}\left[U \tilde{V}_{n} \varphi\left(S_{n}\right) e^{\theta S_{n}} ; M_{n}<0\right]}{\mathbf{E}_{x}\left[e^{\theta S_{n}} ; M_{n}<0\right]} \rightarrow \mathbf{E}_{x}^{-}[U] \mathbf{E}_{\mu_{\theta}}^{+}\left[V \varphi\left(-S_{0}\right)\right] .
$$

Proof. The proofs of both claims are similar. From Proposition 2.1 for $\lambda \geq 0$, $x, y \leq 0$

$$
\frac{\mathbf{E}_{y}\left[e^{(\lambda+\theta) S_{n-j}} ; M_{n-j}<0\right]}{\mathbf{E}_{x}\left[e^{\theta S_{n}} ; M_{n}<0\right]} \rightarrow \frac{v(y) \int_{0}^{\infty} e^{-(\lambda+\theta) z} u(z) d z}{v(x) \int_{0}^{\infty} e^{-\theta z} u(z) d z},
$$

consequently by the continuity theorem for Laplace-transforms for $\varphi: \mathbb{R} \rightarrow \mathbb{R}$, bounded and a.s. continuous with respect to $\mu_{\theta}$

$$
\frac{\mathbf{E}_{y}\left[\varphi\left(S_{n-j}\right) e^{\theta S_{n-j}} ; M_{n-j}<0\right]}{\mathbf{E}_{x}\left[e^{\theta S_{n}} ; M_{n}<0\right]} \rightarrow \frac{v(y)}{v(x)} \int \varphi(-z) \mu_{\theta}(d z) .
$$

In particular this proves the proposition for $i=j=0$. Note that if $\varphi: \mathbb{R} \rightarrow \mathbb{R}$ is positive and a.s. continuous but possibly no longer bounded, we may conclude by a truncation procedure that

$$
\liminf _{n} \frac{\mathbf{E}_{y}\left[\varphi\left(S_{n-j}\right) e^{\theta S_{n-j}} ; M_{n-j}<0\right]}{\mathbf{E}_{x}\left[e^{\theta S_{n}} ; M_{n}<0\right]} \geq \frac{v(y)}{v(x)} \int \varphi(-z) \mu_{\theta}(d z) .
$$

In the general case let us assume without loss of generality $0 \leq g, h \leq 1$. From the Markov property for $n \geq i+j$

$$
\mathbf{E}_{x}\left[U \tilde{V}_{n} \varphi\left(S_{n}\right) e^{\theta S_{n}} ; M_{n}<0\right]=\mathbf{E}_{x}\left[U \psi_{n-i}\left(S_{i}\right) ; M_{i}<0\right]
$$

where for $n \geq j$,

$$
\psi_{n}(y):=\mathbf{E}_{y}\left[\tilde{V}_{n} \varphi\left(S_{n}\right) e^{\theta S_{n}} ; M_{n}<0\right]
$$

By assumption, $\varphi$ is a bounded, continuous function. Thus, discontinuities of $\psi_{j}(y)=\mathbf{E}\left[\tilde{V}_{j} \varphi\left(S_{j}+y\right) e^{\theta\left(S_{j}+y\right)} ; M_{j}<-y\right]$ can only arise from discontinuities 
of $e: y \rightarrow \mathbf{P}\left\{M_{j}<y\right\}$. As bounded, monotone function, $e(\cdot)$ has at most countably many points of discontinuity. Thus the same holds for $\psi_{j}$ and $\psi_{j}$ is a.s. continuous with respect to $\mu_{\theta}$. Therefore it follows from

$$
\psi_{n-i}(y)=\mathbf{E}_{y}\left[\psi_{j}\left(S_{n-i-j}\right) ; M_{n-i-j}<0\right]
$$

and from (2.7)

$$
\liminf _{n} \frac{\psi_{n-i}(y)}{\mathbf{E}_{x}\left[e^{\theta S_{n}} ; M_{n}<0\right]} \geq \frac{v(y)}{v(x)} \int \psi_{j}(-z) e^{\theta z} \mu_{\theta}(d z)
$$

By means of Fatou's Lemma

$$
\begin{aligned}
& \liminf _{n} \mathbf{E}_{x}\left[U \tilde{V}_{n} \varphi\left(S_{n}\right) e^{\theta S_{n}} ; M_{n}<0\right] / \mathbf{E}_{x}\left[e^{\theta S_{n}} ; M_{n}<0\right] \\
& \quad \geq v(x)^{-1} \mathbf{E}_{x}\left[U v\left(S_{i}\right) ; M_{i}<0\right] \cdot \int \psi_{j}(-z) e^{\theta z} \mu_{\theta}(d z) .
\end{aligned}
$$

The first part of the righthand side is equal to $\mathbf{E}_{x}^{-}[U]$. As to the other part we use the duality transformation $Q_{i}^{\prime}:=Q_{j-i+1}, i=1, \ldots, j$ and the corresponding path $S_{1}^{\prime}, \ldots, S_{j}^{\prime}$, the invariance of the Lebesgue measure under the shift transformation $z \mapsto z+S_{j}^{\prime}$ and the fact, that the set $\left\{z: \min \left(S_{0}^{\prime}, \ldots, S_{j-1}^{\prime}\right)=-z\right\}$ has Lebesgue measure 0 and that $S_{0}^{\prime}=0$, to obtain

$$
\begin{aligned}
\int_{0}^{\infty} \psi_{j}(-z) u(z) d z \\
=\int_{0}^{\infty} \mathbf{E}\left[h\left(Q_{1}^{\prime}, \ldots, Q_{j}^{\prime}\right) \varphi\left(S_{j}^{\prime}-z\right) e^{\theta\left(S_{j}^{\prime}-z\right)} ; S_{0}^{\prime}, \ldots, S_{j-1}^{\prime}>S_{j}^{\prime}-z\right] u(z) d z \\
=\mathbf{E} \int_{-S_{j}^{\prime}}^{\infty} h\left(Q_{1}^{\prime}, \ldots, Q_{j}^{\prime}\right) \varphi(-z) e^{-\theta z} 1_{\left\{S_{0}^{\prime} \geq-z\right\}} 1_{\left\{S_{1}^{\prime}, \ldots, S_{j-1}^{\prime} \geq-z\right\}} u\left(S_{j}^{\prime}+z\right) d z \\
=\mathbf{E} \int_{-S_{0}^{\prime}}^{\infty} h\left(Q_{1}^{\prime}, \ldots, Q_{j}^{\prime}\right) \varphi(-z) e^{-\theta z} 1_{\left\{S_{j}^{\prime} \geq-z\right\}} 1_{\left\{S_{1}^{\prime}, \ldots, S_{j-1}^{\prime} \geq-z\right\}} u\left(S_{j}^{\prime}+z\right) d z \\
=\mathbf{E} \int_{0}^{\infty} h\left(Q_{1}^{\prime}, \ldots, Q_{j}^{\prime}\right) \varphi(-z) e^{-\theta z} 1_{\left\{L_{j}^{\prime} \geq-z\right\}} u\left(S_{j}^{\prime}+z\right) d z \\
=\int_{0}^{\infty} \mathbf{E}_{z}\left[h\left(Q_{1}, \ldots, Q_{j}\right) \varphi(-z) u\left(S_{j}\right) ; L_{j} \geq 0\right] e^{-\theta z} d z \\
=\int_{0}^{\infty} \mathbf{E}_{z}^{+}\left[V \varphi\left(-S_{0}\right)\right] u(z) e^{-\theta z} d z .
\end{aligned}
$$

Altogether we end up with the estimate

$$
\liminf _{n} \frac{\mathbf{E}_{x}\left[U \tilde{V}_{n} \varphi\left(S_{n}\right) e^{\theta S_{n}} ; M_{n}<0\right]}{\mathbf{E}_{x}\left[e^{\theta S_{n}} ; M_{n}<0\right]} \geq \mathbf{E}_{x}^{-}[U] \mathbf{E}_{\mu_{\theta}}^{+}\left[V \varphi\left(-S_{0}\right)\right]
$$


Finally replace in this estimate first $g$ by $1-g$ and $h$ by 1 (i.e. $U$ by $1-U$ and $\tilde{V}_{n}, V$ by 1$)$ and second $h$ by $1-h$. Then these estimates altogether entail our claim.

We shall also use a dual version of the last proposition. Let

$$
\tau_{n}:=\min \left\{i \leq n: S_{i}=\min \left(S_{0}, \ldots, S_{n}\right)\right\}
$$

be the moment of the first random walk minimum up to time $n$.

Proposition 2.6. Under the assumptions of Proposition 2.5

$$
\frac{\mathbf{E}\left[U \tilde{V}_{n} \varphi\left(S_{n}\right) e^{\theta S_{n}} ; \tau_{n}=n\right]}{\mathbf{E}\left[e^{\theta S_{n}} ; \tau_{n}=n\right]} \rightarrow \mathbf{E}_{\mu_{\theta}}^{+}\left[U \varphi\left(-S_{0}\right)\right] \mathbf{E}^{-}[V] .
$$

Proof. There is a bounded, measurable function $\psi: \mathcal{D}^{i} \rightarrow \mathbb{R}$ such that a.s.

$$
\psi\left(Q_{1}, \ldots, Q_{i}\right)=\mathbf{E}\left[U \mid Q_{1}, Q_{2}, \ldots, Q_{i}\right] .
$$

By duality for $i+j \leq n$

$$
\begin{aligned}
& \mathbf{E}\left[U \tilde{V}_{n} \varphi\left(S_{n}\right) e^{\theta S_{n}} ; \tau_{n}=n\right] \\
& \quad=\mathbf{E}\left[\psi\left(Q_{1}, \ldots, Q_{i}\right) h\left(Q_{n} \ldots, Q_{n-j+1}\right) \varphi\left(S_{n}\right) e^{\theta S_{n}} ; \tau_{n}=n\right] \\
& \quad=\mathbf{E}\left[h\left(Q_{1}^{\prime}, \ldots, Q_{j}^{\prime}\right) \psi\left(Q_{n}^{\prime}, \ldots, Q_{n-i+1}^{\prime}\right) \varphi\left(S_{n}^{\prime}\right) e^{\theta S_{n}^{\prime}} ; M_{n}^{\prime}<0\right] .
\end{aligned}
$$

Moreover

$$
\mathbf{E}_{\mu_{\theta}}^{+}\left[\psi\left(Q_{1}, \ldots, Q_{i}\right) \varphi\left(-S_{0}\right)\right]=\mathbf{E}_{\mu_{\theta}}^{+}\left[U \varphi\left(-S_{0}\right)\right],
$$

thus the claim follows from the preceding proposition.

The next results on weak convergence generalizing the last propositions are in the spirit of Lemma 2.5 in 4 .

Theorem 2.7. Let $0<\delta<1$. Let $U_{n}=g_{n}\left(R_{0}, \ldots, R_{\lfloor\delta n\rfloor}\right), n \geq 1$, be random variables with values in an Euclidean (or polish) space $S$ such that

$$
U_{n} \rightarrow U_{\infty} \quad \mathbf{P}^{+}-a . s .
$$

for some $S$-valued random variable $U_{\infty}$. Also let $V_{n}=h_{n}\left(Q_{1}, \ldots, Q_{\lfloor\delta n\rfloor}\right), n \geq 1$, be random variables with values in an Euklidean (or polish) space $S^{\prime}$ such that

$$
V_{n} \rightarrow V_{\infty} \quad \mathbf{P}_{x}^{-} \text {-a.s. }
$$


for all $x \leq 0$ and some $S^{\prime}$-valued random variable $V_{\infty}$. Denote

$$
\tilde{V}_{n}:=h_{n}\left(Q_{n}, \ldots, Q_{n-\lfloor\delta n\rfloor+1}\right) .
$$

Then for $\theta>0$ and for any bounded, continuous function $\varphi: S \times S^{\prime} \times \mathbb{R} \rightarrow \mathbb{R}$ as $n \rightarrow \infty$

$$
\begin{aligned}
& \mathbf{E}\left[\varphi\left(U_{n}, \tilde{V}_{n}, S_{n}\right) e^{-\theta S_{n}} ; L_{n} \geq 0\right] / \mathbf{E}\left[e^{-\theta S_{n}} ; L_{n} \geq 0\right] \\
& \rightarrow \iiint \varphi(u, v,-z) \mathbf{P}^{+}\left\{U_{\infty} \in d u\right\} \mathbf{P}_{z}^{-}\left\{V_{\infty} \in d v\right\} \nu_{\theta}(d z) .
\end{aligned}
$$

The following theorem is a counterpart.

Theorem 2.8. Let $U_{n}, V_{n}, \tilde{V}_{n}, n=1,2, \ldots, \infty$ be as in be as in Theorem 2.7. now fulfilling

$$
U_{n} \rightarrow U_{\infty} \quad \mathbf{P}_{x}^{+} \text {-a.s. }, \quad V_{n} \rightarrow V_{\infty} \quad \mathbf{P}^{-} \text {-a.s. }
$$

for all $x \geq 0$. Then for any bounded, continuous function $\varphi: S \times S^{\prime} \times \mathbb{R} \rightarrow \mathbb{R}$ and for $\theta>0$ as $n \rightarrow \infty$

$$
\begin{aligned}
& \mathbf{E}\left[\varphi\left(U_{n}, \tilde{V}_{n}, S_{n}\right) e^{\theta S_{n}} ; \tau_{n}=n\right] / \mathbf{E}\left[e^{\theta S_{n}} ; \tau_{n}=n\right] \\
& \rightarrow \iiint \varphi(u, v,-z) \mathbf{P}_{z}^{+}\left\{U_{\infty} \in d u\right\} \mathbf{P}^{-}\left\{V_{\infty} \in d v\right\} \mu_{\theta}(d z) .
\end{aligned}
$$

The proofs of all three theorems are much the same. We prove the third one.

Proof of Theorem 2.8. The proof relies on two estimates, which allow to switch from $\mathbf{P}$ to $\mathbf{P}^{+}$resp. $\mathbf{P}^{-}$. First we look at the case $\varphi(u, v, z)=\varphi_{1}(u)$, where the function $\varphi_{1}$ is bounded by 1 and depends only on $u$. Then by the Markov property

$$
\mathbf{E}\left[\varphi_{1}\left(U_{n}\right) e^{\theta S_{n}} ; \tau_{n}=n\right]=\mathbf{E}\left[\varphi_{1}\left(U_{n}\right) \psi_{n-\lfloor\delta n\rfloor}\left(S_{\lfloor\delta n\rfloor}, \min \left(S_{0}, \ldots, S_{\lfloor\delta n\rfloor}\right)\right)\right]
$$

with

$$
\psi_{n}(x, y):=\mathbf{E}_{x}\left[e^{\theta S_{n}} ; \tau_{n}=n, S_{n}<y\right] .
$$

By duality and Corollary 2.4 for $x \geq y$

$$
\begin{aligned}
& \psi_{n}(x, y)=e^{\theta x} \mathbf{E}\left[e^{\theta S_{n}} ; \tau_{n}=n, S_{n}<y-x\right] \\
& \quad=e^{\theta x} \mathbf{E}\left[e^{\theta S_{n}} ; M_{n}<0, S_{n}<y-x\right] \leq c b_{n} u(x-y) e^{\theta y},
\end{aligned}
$$


and using $\min \left(S_{0}, \ldots, S_{\lfloor\delta n\rfloor}\right)=L_{\lfloor\delta n\rfloor} \wedge 0$ it follows

$$
\begin{aligned}
& \left|\mathbf{E}\left[\varphi_{1}\left(U_{n}\right) e^{\theta S_{n}} ; \tau_{n}=n\right]\right| \leq \\
& \quad c b_{n-\lfloor\delta n\rfloor} \mathbf{E}\left[\left|\varphi_{1}\left(U_{n}\right)\right| u\left(S_{\lfloor\delta n\rfloor}-L_{\lfloor\delta n\rfloor} \wedge 0\right) e^{\theta L_{\lfloor\delta n\rfloor} \wedge 0}\right] .
\end{aligned}
$$

By martingale property of $u,(2.6)$, we have for any $y \in \mathbb{N}$

$$
\begin{aligned}
& \mathbf{E}\left[u\left(S_{\lfloor\delta n\rfloor}-L_{\lfloor\delta n\rfloor} \wedge 0\right) e^{\theta L_{\lfloor\delta n\rfloor} \wedge 0} ; L_{\lfloor\delta n\rfloor}<-y\right] \\
& \quad \leq \sum_{j=y}^{\infty} e^{-\theta j} \mathbf{E}\left[u\left(S_{\lfloor\delta n\rfloor}+j+1\right) ;-j-1 \leq L_{\lfloor\delta n\rfloor}<-j\right] \\
& \quad \leq \sum_{j=y}^{\infty} e^{-\theta j} \mathbf{E}_{j+1}\left[u\left(S_{\lfloor\delta n\rfloor}\right) ; L_{\lfloor\delta n\rfloor} \geq 0\right] \\
& \quad=\sum_{j=y}^{\infty} e^{-\theta j} u(j+1) .
\end{aligned}
$$

Also by duality and (2.3)

$$
\mathbf{E}\left[e^{\theta S_{n}} ; \tau_{n}=n\right]=\mathbf{E}\left[e^{\theta S_{n}} ; M_{n}<0\right] \sim c b_{n} .
$$

Thus, given $\varepsilon>0$ and choosing $y$ sufficiently large, we have by Proposition 2.1

$$
\begin{aligned}
& \left|\mathbf{E}\left[\varphi_{1}\left(U_{n}\right) e^{\theta S_{n}} ; \tau_{n}=n\right]\right| \leq \varepsilon \mathbf{E}\left[e^{\theta S_{n}} ; \tau_{n}=n\right] \\
& \quad+c b_{n-\lfloor\delta n\rfloor} \mathbf{E}\left[\left|\varphi_{1}\left(U_{n}\right)\right| u\left(S_{\lfloor\delta n\rfloor}+y\right) ; L_{\lfloor\delta n\rfloor} \geq-y\right] .
\end{aligned}
$$

This leads to the estimate

$$
\frac{\left|\mathbf{E}\left[\varphi_{1}\left(U_{n}\right) e^{\theta S_{n}} ; \tau_{n}=n\right]\right|}{\mathbf{E}\left[e^{\theta S_{n}} ; \tau_{n}=n\right]} \leq \varepsilon+c \mathbf{E}_{y}^{+}\left[\left|\varphi_{1}\left(U_{n}\right)\right|\right],
$$

which for given $\varepsilon>0$ holds for $y$ sufficiently large.

Next we look at the case $\varphi(u, v, z)=\varphi_{2}(v)$, where $\varphi_{2}$ again is bounded by 1 . By means of duality and the Markov property we obtain

$$
\begin{aligned}
\mathbf{E}\left[\varphi_{2}\left(\tilde{V}_{n}\right) e^{\theta S_{n}} ; \tau_{n}=n\right]=\mathbf{E}\left[\varphi_{2}\left(V_{n}\right) e^{\theta S_{n}} ; M_{n}<0\right] \\
=\mathbf{E}\left[\varphi_{2}\left(V_{n}\right) \psi_{n-\lfloor\delta n\rfloor}\left(S_{\lfloor\delta n\rfloor}\right) ; M_{\lfloor\delta n\rfloor}<0\right],
\end{aligned}
$$

where

$$
\psi_{n}(x):=\mathbf{E}_{x}\left[e^{\theta S_{n}} ; M_{n}<0\right] .
$$

By means of Corollary 2.4 there is a $c>0$ such that

$$
\left|\mathbf{E}\left[\varphi_{2}\left(V_{n}\right) e^{\theta S_{n}} ; M_{n}<0\right]\right| \leq c b_{n-\lfloor\delta n\rfloor} \mathbf{E}\left[\left|\varphi_{2}\left(V_{n}\right)\right| v\left(S_{\lfloor\delta n\rfloor}\right) ; M_{\lfloor\delta n\rfloor}<0\right] .
$$


Recalling the definition of $\mathbf{E}^{-}$and Proposition 2.1 we obtain for a suitable $c>0$

$$
\left|\frac{\mathbf{E}\left[\varphi_{2}\left(\tilde{V}_{n}\right) e^{\theta S_{n}} ; \tau_{n}=n\right]}{\mathbf{E}\left[e^{\theta S_{n}} ; \tau_{n}=n\right]}\right| \leq c \mathbf{E}^{-}\left[\left|\varphi_{2}\left(V_{n}\right)\right|\right] .
$$

Now denote

$$
\tilde{V}_{k, n}:=h_{k}\left(Q_{n}, \ldots, Q_{n-\lfloor\delta k\rfloor+1}\right) .
$$

If $\varphi_{3}$ depends only on $z$ and is continuous and bounded by 1 , then we obtain by means of (2.10) and (2.11) (replacing $\varphi_{1}\left(U_{n}\right)$ and $\varphi_{2}\left(\tilde{V}_{n}\right)$ by $\varphi_{1}\left(U_{n}\right)-\varphi_{1}\left(U_{k}\right)$ and $\varphi_{2}\left(\tilde{V}_{n}\right)-\varphi_{2}\left(\tilde{V}_{k, n}\right)$ in these estimates)

$$
\begin{aligned}
& \frac{\left|\mathbf{E}\left[\left(\varphi_{1}\left(U_{n}\right) \varphi_{2}\left(\tilde{V}_{n}\right)-\varphi_{1}\left(U_{k}\right) \varphi_{2}\left(\tilde{V}_{k, n}\right)\right) \varphi_{3}\left(S_{n}\right) e^{\theta S_{n}} ; \tau_{n}=n\right]\right|}{\mathbf{E}\left[e^{\theta S_{n}} ; \tau_{n}=n\right]} \\
& \quad \leq \frac{\mathbf{E}\left[\left|\varphi_{1}\left(U_{n}\right)-\varphi_{1}\left(U_{k}\right)\right| e^{\theta S_{n}} ; \tau_{n}=n\right]}{\mathbf{E}\left[e^{\theta S_{n}} ; \tau_{n}=n\right]}+\frac{\mathbf{E}\left[\left|\varphi_{2}\left(\tilde{V}_{n}\right)-\varphi_{2}\left(\tilde{V}_{k, n}\right)\right| e^{\theta S_{n}} ; \tau_{n}=n\right]}{\mathbf{E}\left[e^{\theta S_{n}} ; \tau_{n}=n\right]} \\
& \quad \leq c \mathbf{E}_{y}^{+}\left[\left|\varphi_{1}\left(U_{n}\right)-\varphi_{1}\left(U_{k}\right)\right|\right]+\varepsilon+c \mathbf{E}^{-}\left[\left|\varphi_{2}\left(V_{n}\right)-\varphi_{2}\left(V_{k}\right)\right|\right],
\end{aligned}
$$

if $c, y$ are sufficiently large. Letting $n \rightarrow \infty$ we obtain by assumption and Proposition 2.6

$$
\begin{aligned}
& \limsup _{n} \mid \frac{\mathbf{E}\left[\left(\varphi_{1}\left(U_{n}\right) \varphi_{2}\left(\tilde{V}_{n}\right) \varphi_{3}\left(S_{n}\right) e^{\theta S_{n}} ; \tau_{n}=n\right]\right.}{\mathbf{E}\left[e^{\theta S_{n}} ; \tau_{n}=n\right]} \\
&-\mathbf{E}_{\mu_{\theta}}^{+}\left[\varphi_{1}\left(U_{k}\right) \varphi_{3}\left(-S_{0}\right)\right] \mathbf{E}^{-}\left[\varphi_{2}\left(V_{k}\right)\right] \mid \\
& \leq \varepsilon+c \mathbf{E}_{y}^{+}\left[\left|\varphi_{1}\left(U_{\infty}\right)-\varphi_{1}\left(U_{k}\right)\right|\right]+c \mathbf{E}^{-}\left[\left|\varphi_{2}\left(V_{\infty}\right)-\varphi_{2}\left(V_{k}\right)\right|\right]
\end{aligned}
$$

Also by assumption the terms on the righthand side vanish for $k \rightarrow \infty$. Letting $\varepsilon \rightarrow 0$, our claim follows in the case $\varphi(u, v, z)=\varphi_{1}(u) \varphi_{2}(v) \varphi_{3}(z)$. As is wellknown this case is sufficient for the proof of weak convergence.

\section{Proof of theorems}

Define

$$
\eta_{i}:=\sum_{y=0}^{\infty} y(y-1) Q_{i}(\{y\}) /\left(\sum_{y=0}^{\infty} y Q_{i}(\{y\})\right)^{2}, \quad i \geq 1 .
$$

Lemma 3.1. Assume A2 and A3. Then for all $x \geq 0$

$$
\sum_{i=0}^{\infty} \eta_{i+1} e^{-S_{i}}<\infty \quad \mathbf{P}_{x}^{+}-\text {a.s. }
$$


and for all $x \leq 0$

$$
\sum_{i=1}^{\infty} \eta_{i} e^{S_{i}}<\infty \quad \mathbf{P}_{x}^{-}-\text {a.s. }
$$

The proof of the first statement can be found in 4] (see Lemma 2.7 therein under condition B1 and B2), the second one can be proven just the same way.

The branching mechanism can be neatly described by means of generating functions. Let

$$
f_{j}(s):=\sum_{i=0}^{\infty} s^{i} Q_{j}(\{i\}), \quad 0 \leq s \leq 1,
$$

$j=1,2, \ldots$ and their compositions

$$
\begin{aligned}
f_{k, n} & :=f_{k+1}\left(f_{k+2}\left(\cdots f_{n}(s) \cdots\right)\right), \quad 0 \leq k<n, \\
f_{k, 0} & :=f_{k}\left(f_{k-1}\left(\cdots f_{1}(s) \cdots\right)\right), \quad 0<k .
\end{aligned}
$$

As is well-known the branching property can be expressed as

$$
\mathbb{E}\left[s^{Z_{n}} \mid \Pi, Z_{k}\right]=f_{k, n}(s)^{Z_{k}} \quad \mathbb{P} \text {-a.s. }
$$

and it also holds after a change of measure and conditioning (compare section 3 in [4]),

$$
\mathbf{E}^{ \pm}\left[s^{Z_{n}} \mid \Pi, Z_{k}\right]=f_{k, n}(s)^{Z_{k}} \quad \mathbf{P}^{ \pm} \text {-a.s. }
$$

We shall use the following fact.

Lemma 3.2. For any $0 \leq s \leq 1$ the sequence $f_{k, 0}(s)^{\exp \left(-S_{k}\right)}, k \geq 1$, is nondecreasing and $f_{k, 0}(s)^{\exp \left(-S_{k}\right)} \geq s^{\exp \left(-S_{0}\right)}$.

Proof. Without loss of generality assume $s>0$. We use the fact that the cumulant generating functions $c_{k}(\lambda):=\log f_{k}\left(e^{\lambda}\right), \lambda \leq 0$, are convex. Since $c_{k}(0)=0$, this implies $c_{k}(\lambda) \geq c_{k}^{\prime}(0) \lambda$ or, letting $\lambda=\log t, 0<t \leq 1$,

$$
\log f_{k}(t) \geq f_{k}^{\prime}(1) \log t=e^{X_{k}} \log t .
$$

Choosing $t=f_{k-1,0}(s)$ and multiplying with $\exp \left(-S_{k}\right)$ gives the first statement. For $k=1$ the second inequality follows.

Under the measure $\mathbb{P}$, Proposition 2.1 translates to 
Corollary 3.3. For $x \geq 0, \theta>-\beta$

$$
\mathbb{E}_{x}\left[e^{-\theta S_{n}} ; L_{n} \geq 0\right] \sim s(0) \gamma^{n} b_{n} u(x) e^{\beta x} \int_{0}^{\infty} e^{-(\theta+\beta) z} v(-z) d z,
$$

and for $x \leq 0$ and $\theta>\beta$

$$
\mathbb{E}_{x}\left[e^{\theta S_{n}} ; M_{n}<0\right] \sim s(0) \gamma^{n} b_{n} v(x) e^{\beta x} \int_{0}^{\infty} e^{-(\theta-\beta) z} u(z) d z
$$

Proof. We only prove the second statement. Let $x \leq 0$ and $\theta>\beta$. By the usual change of measure,

$$
\begin{gathered}
\mathbb{E}_{x}\left[e^{\theta S_{n}} ; M_{n}<0\right]=\gamma^{n} e^{\theta x} \mathbf{E}\left[e^{(\theta-\beta) S_{n}} ; M_{n}<-x\right] \\
\quad=\gamma^{n} e^{\beta x} \mathbf{E}_{x}\left[e^{(\theta-\beta) S_{n}} ; M_{n}<0\right]
\end{gathered}
$$

and the result follows from Proposition 2.1

With the corresponding change to $\theta=\vartheta+\beta$, in the following, Theorem 2.7 and 2.8 will be used. By the change of measure used before, the theorems can be applied to the measure $\mathbb{P}$. For later use we note that

$$
\mathbb{E}\left[e^{S_{n}} ; \tau_{n}=n\right] \sim c \mathbb{P}\left\{L_{n} \geq 0\right\}
$$

with some number $c>0$, which follows from the last corollary together with (2.9).

Lemma 3.4. Let $z \geq 1$ and let $m_{n}, n \geq 1$, be a sequence of natural numbers with $m_{n} \sim n / 2$. Then, as $n \rightarrow \infty$, the random vector $\left(\exp \left(-S_{m_{n}}\right) Z_{m_{n}}, Z_{n}\right)$, given the event $\left\{Z_{0}=z, L_{n} \geq 0\right\}$, converges in distribution to some random vector $(W, G)$ with values in $[0, \infty) \times \mathbb{N}_{0}$. Moreover the probability of $G \geq 1$ is greater than 0 and $W>0$ a.s. on the event $G \geq 1$.

Proof. We prove convergence of $\mathbb{E}\left[U_{n}^{\prime} s^{Z_{n}} \mid Z_{0}=z, L_{n} \geq 0\right]$ for $0<s \leq 1$ and suitable bounded random variables $U_{n}^{\prime}$. Define

$$
\varphi\left(u^{\prime}, u^{\prime \prime}, v, x\right):=u^{\prime} v^{u^{\prime \prime} \exp (x)}, \quad \text { for } 0 \leq u^{\prime} \leq 1, u^{\prime \prime} \geq 0,0 \leq v \leq 1, x \in \mathbb{R},
$$

with $0^{0}=1$. For other values of $\left(u^{\prime}, u^{\prime \prime}, v, x\right)$ let $\varphi\left(u^{\prime}, u^{\prime \prime}, v, x\right)$ be such that $\varphi$ becomes a bounded, continuous function. In doing so points of discontinuity in $\left(u^{\prime}, 0,0, x\right)$ are unavoidable, which will be bypassed in the sequel. Moreover let

$$
\begin{aligned}
U_{n}=\left(U_{n}^{\prime}, U_{n}^{\prime \prime}\right) & :=\left(U_{n}^{\prime}, \exp \left(-S_{m_{n}}\right) Z_{m_{n}}\right), \\
V_{n} & :=f_{n-m_{n}, 0}(s)^{\exp \left(-S_{n-m_{n}}\right)},
\end{aligned}
$$


and thus $\tilde{V}_{n}=f_{m_{n}, n}(s)^{\exp \left(-\left(S_{n}-S_{m_{n}}\right)\right)}$. If we assume that $U_{n}^{\prime}$ is a random variable with values between 0 and 1 of the form $U_{n}^{\prime}=h\left(S_{m_{n}}, Z_{m_{n}}\right)$, then

$$
\mathbb{E}\left[U_{n}^{\prime} s^{Z_{n}} \mid \Pi, Z_{m_{n}}\right]=U_{n}^{\prime} f_{m_{n}, n}(s)^{Z_{m_{n}}}=\varphi\left(U_{n}, \tilde{V}_{n}, S_{n}\right)
$$

and

$$
\mathbb{E}\left[U_{n}^{\prime} s^{Z_{n}} \mid Z_{0}=z, L_{n} \geq 0\right]=\mathbb{E}\left[\varphi\left(U_{n}, \tilde{V}_{n}, S_{n}\right) \mid Z_{0}=z, L_{n} \geq 0\right] .
$$

We would like to apply Theorem 2.7. From Lemma 3.2 it follows that $V_{n}$ converges to some random variable $V_{\infty}$ such that $0<s^{\exp \left(-S_{0}\right)} \leq V_{\infty} \leq 1$. From Proposition 3.1 in [4] (with the Assumptions B1 and B2 therein) we see that $U_{n}^{\prime \prime}$ converges $\mathbf{P}^{+}$-a.s. to a random variable $U_{\infty}^{\prime \prime}$. Also $\mathbf{P}^{+}\left(U_{\infty}^{\prime \prime}>0\right)>0$. Thus we just have to take care that $U_{n}^{\prime}$ converges $\mathbf{P}^{+}$-a.s. to some random variable $U_{\infty}^{\prime}$

Now $\varphi$ is continuous in every point $\left(u^{\prime}, u^{\prime \prime}, v, x\right)$ with $v>0$, thus we conclude from Theorem 2.7 and standard results on weak convergence that

$$
\mathbb{E}\left[U_{n}^{\prime} s^{Z_{n}} \mid Z_{0}=z, L_{n} \geq 0\right] \rightarrow \psi_{z}(s) \quad, 0<s \leq 1,
$$

where

$$
\psi_{z}(s):=\iiint \varphi(u, v,-x) \mathbf{P}^{+}\left\{U_{\infty} \in d u\right\} \mathbf{P}_{x}^{-}\left\{V_{\infty} \in d v\right\} \nu_{\beta}(d x),
$$

and $u=\left(u^{\prime}, u^{\prime \prime}\right)$ and $U_{\infty}=\left(U_{\infty}^{\prime}, U_{\infty}^{\prime \prime}\right)$. Note that the distribution of $U_{\infty}$ depends only on $z$ whereas the distribution of $V_{\infty}$ depends on $s$. From $s^{\exp \left(-S_{0}\right)} \leq V_{\infty} \leq$ 1 it follows, that

$$
\psi_{z}(s) \rightarrow \psi_{z}(1)=\mathbf{E}^{+}\left[U_{\infty}^{\prime}\right], \text { as } s \rightarrow 1 .
$$

First let us choose $U_{n}^{\prime}=1$ for all $n$. Then for $0<s \leq 1$ we obtain convergence of the generating function $\mathbb{E}\left[s^{Z_{n}} \mid Z_{0}=z, L_{n} \geq 0\right]$ to some function $\psi_{z}(s)$ with $\psi_{z}(s) \rightarrow 1$, as $s \rightarrow 1$. Thus $\mathcal{L}\left(Z_{n} \mid Z_{0}=z, L_{n} \geq 0\right)$ is weakly convergent to some probability measure with generating function $\psi_{z}$. In order to show that this measure is not the Dirac measure at 0 we prove that $V_{\infty}<1 \mathbf{P}_{x}^{-}$-a.s. for $s<1$. To this end we use

$$
-\log V_{n} \geq \exp \left(-S_{n-m_{n}}\right)\left(1-f_{n-m_{n}, 0}(s)\right)
$$

together with an estimate for $f_{k, n}$ due to Agresti [6] (see also the proof of Proposition 3.1 in [4]), which for $f_{k, 0}$ reads

$$
\exp \left(-S_{k}\right)\left(1-f_{k, 0}(s)\right) \geq\left(\frac{1}{1-s}+\sum_{i=1}^{k} \eta_{i} e^{S_{i}}\right)^{-1} .
$$


From Lemma 3.1 we see that $-\log V_{\infty}>0$ and thus $V_{\infty}<1 \mathbf{P}_{x}^{-}$-a.s. Also, as already mentioned, $\mathbf{P}^{+}\left(U_{\infty}^{\prime \prime}>0\right)>0$. By definition of $\varphi(u, v, x)$ this implies $\psi_{z}(s)<1$ for $s<1$. Therefore the corresponding probability measure is not concentrated at 0 .

Next we choose $s=1\left(\right.$ thus $\left.V_{n}=1\right)$ and $U_{n}^{\prime}:=\chi\left(\exp \left(-S_{m_{n}}\right) Z_{m_{n}}\right)=\chi\left(U_{n}^{\prime \prime}\right)$, where $\chi: \mathbb{R} \rightarrow[0,1]$ denotes a continuous function. Then by (3.4), we obtain that

$$
\mathbb{E}\left[\chi\left(\exp \left(-S_{m_{n}}\right) Z_{m_{n}}\right) \mid Z_{0}=z, L_{n} \geq 0\right] \rightarrow \mathbf{E}^{+}\left[\chi\left(U_{\infty}^{\prime \prime}\right)\right] .
$$

This gives weak convergence of $\mathcal{L}\left(\exp \left(-S_{m_{n}}\right) Z_{m_{n}} \mid Z_{0}=z, L_{n} \geq 0\right)$ to some probability measure on $\mathbb{R}^{+}$. The convergence in (3.4) also implies that

$$
\mathbb{E}\left[\chi\left(\exp \left(-S_{m_{n}}\right) Z_{m_{n}}\right) s^{Z_{n}} \mid Z_{0}=z, L_{n} \geq 0\right]
$$

has a limit for any $0<s \leq 1$ and any bounded continuous $\chi$. Therefore, given the event $\left\{Z_{0}=z, L_{n} \geq 0\right\}$, the joint distribution of $\exp \left(-S_{m_{n}}\right) Z_{m_{n}}$ and $Z_{n}$ is weakly convergent, too. We write the limiting distribution as the distribution of some pair $(W, G)$ of random variables with values in $\mathbb{R}^{+} \times \mathbb{N}_{0}$ and, for ease of notation, we denote the corresponding probabilities and expectations by $\mathbf{P}$ and E. We already proved that $\mathbf{P}\{G \geq 1\}>0$.

For the last claim of the lemma we use our convergence result for $s=1$ and $U_{n}^{\prime}:=I_{\left\{Z_{m_{n}} \geq 1\right\}} \chi\left(\exp \left(-S_{m_{n}}\right) Z_{m_{n}}\right)$ with continuous $\chi$ with values in [0,1]. From Proposition 3.1 in [4] it follows that $U_{n}^{\prime}$ converges to $I_{\left\{U_{\infty}^{\prime \prime}>0\right\}} \chi\left(U_{\infty}^{\prime \prime}\right) \mathbf{P}^{+}$-a.s. and consequently

$$
\mathbb{E}\left[I_{\left\{Z_{m_{n}} \geq 1\right\}} \chi\left(\exp \left(-S_{m_{n}}\right) Z_{m_{n}}\right) \mid Z_{0}=z, L_{n} \geq 0\right] \rightarrow \mathbf{E}\left[I_{\{W>0\}} \chi(W)\right] .
$$

On the other hand we know that

$$
\mathbb{E}\left[I_{\left\{Z_{n} \geq 1\right\}} \chi\left(\exp \left(-S_{m_{n}}\right) Z_{m_{n}}\right) \mid Z_{0}=z, L_{n} \geq 0\right] \rightarrow \mathbf{E}[\chi(W) ; G \geq 1] .
$$

Now $I_{\left\{Z_{n} \geq 1\right\}} \leq I_{\left\{Z_{\left.m_{n} \geq 1\right\}}\right.}$, therefore $\mathbf{E}[\chi(W) ; G \geq 1] \leq \mathbf{E}\left[I_{\{W>0\}} \chi(W)\right]$. For $\eta>0$ (choosing an appropriate $\chi$ ) it follows that

$$
\mathbf{P}\{W=0, G \geq 1\} \leq \mathbf{P}\{0<W \leq \eta\} .
$$

Letting $\eta \rightarrow 0$, this gives $\mathbf{P}\{W=0, G \geq 1\}=0$, which is our last claim.

Lemma 3.5. Let $m_{n}, n \geq 1$, be such that $m_{n} \sim n / 2$ and $\tau_{n}$ be defined as in (2.8). Then the conditional distribution $\mathcal{L}\left(\left(\exp \left(-S_{m_{n}}\right) Z_{m_{n}}, Z_{n}\right) \mid Z_{n}>0, \tau_{n}=\right.$ 
$n)$ converges to some random vector $(W, G)$ with values in $(0, \infty) \times \mathbb{N}$. Moreover there is a number $0<\kappa<\infty$ such that

$$
\mathbb{P}\left\{Z_{n}>0, \tau_{n}=n\right\} \sim \kappa \mathbb{P}\left\{L_{n} \geq 0\right\}
$$

Proof. The proof is somewhat different from the preceding one. For $a>0$ let

$$
\varphi_{a}\left(u^{\prime}, u^{\prime \prime}, v, x\right):=u^{\prime}\left(1-v^{u^{\prime \prime} \exp (x)}\right) 1_{\{x \geq-a\}} e^{-x},
$$

for $0 \leq u^{\prime} \leq 1, u^{\prime \prime} \geq 0,0 \leq v \leq 1, x \in \mathbb{R}$ and continue $\varphi_{a}$ to other values of $u^{\prime}, u^{\prime \prime}, v, x$ to a bounded, smooth function. Conditioning as above we obtain

$$
\mathbb{E}\left[U_{n}^{\prime}\left(1-s^{Z_{n}}\right) 1_{\left\{S_{n} \geq-a\right\}} ; \tau_{n}=n\right]=\mathbb{E}\left[\varphi_{a}\left(U_{n}, \tilde{V}_{n}, S_{n}\right) e^{S_{n}} ; \tau_{n}=n\right],
$$

where $U_{n}=\left(U_{n}^{\prime}, U_{n}^{\prime \prime}\right), \tilde{V}_{n}$ are as in the last proof. Note, that the additional discontinuity at $x=-a$ has probability 0 with respect to the measure $\mu_{1-\beta}$. Thus we may apply Theorem 2.8 to $\varphi_{a}(u, v, x)$.

Moreover, as $1-s^{Z_{n}} \leq Z_{n}$ and by duality

$$
\begin{aligned}
\mathbb{E}\left[\left|U_{n}^{\prime}\right|\left(1-s^{Z_{n}}\right) 1_{\left\{S_{n}<-a\right\}} ; \tau_{n}=n\right] \leq \mathbb{E}\left[Z_{n} ; S_{n}<-a, \tau_{n}=n\right] \\
=\mathbb{E}\left[e^{S_{n}} ; S_{n}<-a, \tau_{n}=n\right]=\mathbb{E}\left[e^{S_{n}} ; S_{n}<-a, M_{n}<0\right] .
\end{aligned}
$$

In view of (2.4) (translated to the measure $\mathbb{P}$ by the usual tilting), there is for every $\varepsilon>0$ an $a>0$ such that

$$
\mathbb{E}\left[1-s^{Z_{n}} ; S_{n}<-a, \tau_{n}=n\right] \leq \varepsilon \mathbb{E}\left[e^{S_{n}} ; \tau_{n}=n\right]
$$

for all $n$. Hence we conclude that the statement of Theorem 2.8 holds for $\varphi_{\infty}(u, v, x)$, too, and we obtain as in the preceding proof

$$
\hat{\psi}_{n}(s):=\mathbb{E}\left[U_{n}^{\prime}\left(1-s^{Z_{n}}\right) ; \tau_{n}=n\right] / \mathbb{E}\left[e^{S_{n}} ; \tau_{n}=n\right] \rightarrow \hat{\psi}(s)
$$

for $0<s \leq 1$, with

$$
\hat{\psi}(s):=\iiint u^{\prime}\left(1-v^{u^{\prime \prime} \exp (x)}\right) e^{x} \mathbf{P}_{x}^{+}\left\{U_{\infty} \in d u\right\} \mathbf{P}^{-}\left\{V_{\infty} \in d v\right\} \mu_{1-\beta}(d x) .
$$

First we note that $\hat{\psi}(s)$ is right continuous at 0 : With decreasing $s>0$ also the values of $V_{\infty}$ decrease, and the integrand of $\hat{\psi}(s)$ increases. Also for $u^{\prime}, u^{\prime \prime}, x$ fixed the integrand is continuous in $s$ (with $0^{0}=1$ ). Therefore monotone convergence implies $\hat{\psi}(0+)=\hat{\psi}(0)$. 
Next we note that the functions $\hat{\psi}_{n}(s), n \geq 1$, are uniformly bounded analytical functions on the complex unit disc and convergent for $0<s<1$. As is well known this implies convergence of $\hat{\psi}_{n}(s)$ to an analytic function on the unit disc. In particular $\hat{\psi}_{n}(0) \rightarrow \hat{\psi}(0)$, since $\hat{\psi}(0+)=\hat{\psi}(0)$. Also this convergence implies that the coefficients of the power series $\hat{\psi}_{n}(s)$, namely

$$
\frac{\mathbb{E}\left[U_{n}^{\prime} ; Z_{n}>0, \tau_{n}=n\right]}{\mathbb{E}\left[e^{S_{n}} ; \tau_{n}=n\right]} \text { and } \frac{\mathbb{E}\left[U_{n}^{\prime} ; Z_{n}=k, \tau_{n}=n\right]}{\mathbb{E}\left[e^{S_{n}} ; \tau_{n}=n\right]} \text { with } k \geq 1
$$

are convergent for $n \rightarrow \infty$.

Now let us look at the case $U_{n}^{\prime}=1$. Then we obtain the existence of the limits

$$
\kappa_{0}=\lim _{n \rightarrow \infty} \frac{\mathbb{P}\left\{Z_{n}>0, \tau_{n}=n\right\}}{\mathbb{E}\left[e^{S_{n}} ; \tau_{n}=n\right]} \quad \text { and } \quad \lim _{n \rightarrow \infty} \frac{\mathbb{P}\left\{Z_{n}=k, \tau_{n}=n\right\}}{\mathbb{E}\left[e^{S_{n}} ; \tau_{n}=n\right]}
$$

for $k \geq 1$. Also $\hat{\psi}(s)>0$ for $s<1$, which follows exactly as $\psi_{z}(s)<1$ in the proof of the last lemma. This implies $\kappa_{0}>0$, which, together with (3.3), gives the last statement of the lemma. Also it follows that

$$
\lim _{n \rightarrow \infty} \mathbb{P}\left\{Z_{n}=k \mid Z_{n}>0, \tau_{n}=n\right\}
$$

exists for all $k \geq 1$. We have to verify that the limiting measure is a probability distribution, that is we have to prove that the sequence of conditional distributions of $Z_{n}$, given $\left\{Z_{n}>0, \tau_{n}=n\right\}$, is tight. This follows from the estimate

$$
\begin{aligned}
\mathbb{P}\left\{Z_{n}>k, \tau_{n}=n\right\} & \leq \frac{1}{k} \mathbb{E}\left[Z_{n} ; \tau_{n}=n\right]=\frac{1}{k} \mathbb{E}\left[e^{S_{n}} ; \tau_{n}=n\right] \\
& \sim \frac{1}{k} \frac{\mathbb{P}\left\{Z_{n}>0 ; \tau_{n}=n\right\}}{\kappa_{0}} .
\end{aligned}
$$

Thus all statements on $Z_{n}$ are proven.

Next we consider convergence of the conditional distribution $U_{n}^{\prime \prime}=$ $\exp \left(-S_{m_{n}}\right) Z_{m_{n}}$. For this purpose let $U_{n}^{\prime}=I_{\left\{Z_{\left.m_{n} \geq 1\right\}}\right.} \chi\left(U_{n}^{\prime \prime}\right)$ with $\chi$ continuous and bounded with values between 0 and 1. From Proposition 3.1 in [4 $U_{n}^{\prime}$ converges to $I_{\left\{U_{\infty}^{\prime \prime}>0\right\}} \chi\left(U_{\infty}^{\prime \prime}\right) \mathbf{P}_{x}^{+}$-a.s. for all $x$. Since $Z_{n}>0$ implies $Z_{m_{n}}>0$

$$
\frac{\mathbb{E}\left[\chi\left(U_{n}^{\prime \prime}\right) ; Z_{n}>0, \tau_{n}=n\right]}{\mathbb{E}\left[e^{S_{n}} ; \tau_{n}=n\right]}=\frac{\mathbb{E}\left[U_{n}^{\prime} ; Z_{n}>0, \tau_{n}=n\right]}{\mathbb{E}\left[e^{S_{n}} ; \tau_{n}=n\right]}=\hat{\psi}_{n}(0) .
$$

From $\hat{\psi}_{n}(0) \rightarrow \hat{\psi}(0)$ and from the definition of $\kappa_{0}$ it follows

$$
\begin{aligned}
\mathbb{E}\left[\chi\left(U_{n}^{\prime \prime}\right) ;\right. & \left.Z_{n}>0, \tau_{n}=n\right] / \mathbb{P}\left\{Z_{n}>0, \tau_{n}=n\right\} \\
& \rightarrow \frac{1}{\kappa_{0}} \iiint 1_{\left\{u^{\prime \prime}>0\right\}} \chi\left(u^{\prime \prime}\right)\left(1-v^{u^{\prime \prime} \exp (x)}\right) e^{x} \pi\left(d u^{\prime \prime}, d v, d x\right)
\end{aligned}
$$


with $\pi\left(d u^{\prime \prime}, d v, d x\right)=\mathbf{P}_{x}^{+}\left\{U_{\infty}^{\prime \prime} \in d u^{\prime \prime}\right\} \mathbf{P}^{-}\left\{V_{\infty} \in d v\right\} \mu_{1-\beta}(d x)$. This implies weak convergence of the distribution of $U_{n}^{\prime \prime}$, given $\left\{Z_{n}>0, \tau_{n}=n\right\}$, to a probability distribution. Also, because of the appearence of $1_{\left\{u^{\prime \prime}>0\right\}}$ in the integral this distribution is concentrated on $(0, \infty)$. Finally we also have the convergence of the coefficients of the power series $\hat{\psi}_{n}(s)$ and consequently the existence of the limits

$$
\lim _{n \rightarrow \infty} \frac{\mathbb{E}\left[\chi\left(U_{n}^{\prime \prime}\right) ; Z_{n}=k, \tau_{n}=n\right\}}{\mathbb{P}\left\{Z_{n}>0, \tau_{n}=n\right\}}
$$

for $k \geq 1$. This implies convergence of the joint distribution of $\exp \left(-S_{m_{n}}\right) Z_{m_{n}}$ and $Z_{n}$, given $\left\{Z_{n}>0, \tau_{n}=n\right\}$.

Lemma 3.6. Under $A 1$ to $A 3$, for every $B \subset \mathbb{N}=\{1,2, \ldots\}$ there exists $0 \leq \kappa(B)<\infty$ such that

$$
\frac{\mathbb{P}\left\{Z_{n} \in B\right\}}{\mathbb{P}\left\{L_{n} \geq 0\right\}} \rightarrow \kappa(B)
$$

Also $\kappa>0$ for $B=\mathbb{N}$.

Proof. We decompose at the moment, when $S_{1}, \ldots, S_{n}$ takes its minimum for the first time:

$$
\mathbb{P}\left\{Z_{n} \in B\right\}=\sum_{k=0}^{n} \mathbb{P}\left\{Z_{n} \in B, \tau_{k}=k, \min _{k<l \leq n} S_{l} \geq S_{k}\right\} .
$$

Letting

$$
\xi_{n}(z)=\mathbb{P}\left\{Z_{n} \in B \mid Z_{0}=z, L_{n} \geq 0\right\}
$$

we obtain for fixed $m \geq 1$ and $n \geq 2 m$

$$
\begin{aligned}
\mathbb{P}\left\{Z_{n} \in B\right\}= & \sum_{k=0}^{m-1} \mathbb{E}\left[\xi_{n-k}\left(Z_{k}\right) ; \tau_{k}=k\right] \mathbb{P}\left\{L_{n-k} \geq 0\right\} \\
& +\sum_{k=m}^{n-m} \mathbb{E}\left[\xi_{n-k}\left(Z_{k}\right) ; \tau_{k}=k\right] \mathbb{P}\left\{L_{n-k} \geq 0\right\} \\
& +\sum_{j=0}^{m-1} \mathbb{E}\left[\xi_{j}\left(Z_{n-j}\right) ; \tau_{n-j}=n-j\right] \mathbb{P}\left\{L_{j} \geq 0\right\} .
\end{aligned}
$$

As to the sum in (3.5), $\xi_{n}(z)$ is bounded by 1 and in view of Lemma 3.4 converges for every $z \geq 1$. Also $\mathbb{P}\left\{L_{n-k} \geq 0\right\} \sim \gamma^{-k} \mathbb{P}\left\{L_{n} \geq 0\right\}$ by 
Corollary 3.3. Therefore there is a number $0 \leq \kappa^{\prime}<\infty$ (depending on $m$ and $B$ ) such that

$$
\sum_{k=0}^{m-1} \mathbb{E}\left[\xi_{n-k}\left(Z_{k}\right) ; \tau_{k}=k\right] \mathbb{P}\left\{L_{n-k} \geq 0\right\}=\left(\kappa^{\prime}+o(1)\right) \mathbb{P}\left\{L_{n} \geq 0\right\} .
$$

The sum in (3.6) may be estimated from above by

$$
\sum_{k=m}^{n-m} \mathbb{P}\left\{Z_{k}>0, \tau_{k}=k\right\} \mathbb{P}\left\{L_{n-k} \geq 0\right\}
$$

or in view of Lemma 3.5 by

$$
\sum_{k=m}^{n-m} \mathbb{P}\left\{L_{k} \geq 0\right\} \mathbb{P}\left\{L_{n-k} \geq 0\right\}
$$

up to some factor independent of $m$. In view of Corollary 3.3 this may be bounded by

$$
\gamma^{n} \sum_{k=m}^{n-m} b_{k} \cdot b_{n-k},
$$

again up to an uniform factor. Lemma 2.2 shows that this quantity is asymptotically equal to $\gamma^{n} b_{n} 2 \sum_{k=m}^{\infty} b_{k}$. Altogether, in view of Corollary 3.3 for any $\varepsilon>0$

$$
\sum_{k=m}^{n-m} \mathbb{E}\left[\xi_{n-k}\left(Z_{n-k}\right) ; \tau_{k}=k\right] \mathbb{P}\left\{L_{n-k} \geq 0\right\} \leq \varepsilon \mathbb{P}\left\{L_{n} \geq 0\right\},
$$

if only $m$ is large enough.

Finally for the sum in (3.7)

$$
\begin{aligned}
& \mathbb{E}\left[\xi_{j}\left(Z_{n-j}\right) ; \tau_{n-j}=n-j\right] \\
& \quad=\mathbb{E}\left[\xi_{j}\left(Z_{n-j}\right) \mid Z_{n-j}>0, \tau_{n-j}=n-j\right] \mathbb{P}\left\{Z_{n-j}>0, \tau_{n-j}=n-j\right\} .
\end{aligned}
$$

The first term in righthand-side of the above equation is bounded by 1 and it follows with Lemma 3.5 that

$$
\sum_{j=0}^{m-1} \mathbb{E}\left[\xi_{j}\left(Z_{n-j}\right) ; \tau_{n-j}=n-j\right] \mathbb{P}\left\{L_{j} \geq 0\right\}=\left(\kappa^{\prime \prime}+o(1)\right) \mathbb{P}\left\{L_{n} \geq 0\right\}
$$

for some $\kappa^{\prime \prime} \geq 0$.

Altogether, letting $m \rightarrow \infty$, these three statements imply the first claim of the lemma. Also, if $B=\mathbb{N}$, then because of Lemma 3.4 the limit of $\xi_{n}(z)$ is strictly positive for all $z$. Because of Lemma $3.5 \kappa^{\prime}>0$ for $m$ sufficiently large. This gives the second statement. 
Proof of Theorem 1.1. This theorem is contained in the last lemma.

Proof of Theorem 1.3, First we estimate $\mathbb{E}\left[Z_{n}^{\vartheta}\right]$ :

$$
\begin{aligned}
\mathbb{E}\left[Z_{n}^{\vartheta}\right] & =\sum_{k=0}^{n} \mathbb{E}\left[Z_{n}^{\vartheta} ; \tau_{k}=k, \min _{k<l \leq n} S_{l} \geq S_{k}\right] \\
& =\sum_{k=0}^{n} \mathbb{E}\left[\eta_{n-k}\left(Z_{k}\right) ; \tau_{k}=k\right] \mathbb{P}\left\{L_{n-k} \geq 0\right\}
\end{aligned}
$$

with

$$
\eta_{n}(z):=\mathbb{E}\left[Z_{n}^{\vartheta} \mid Z_{0}=z, L_{n} \geq 0\right] .
$$

From Jensen's inequality for $\vartheta<1$

$$
\begin{aligned}
\eta_{n}(z) & \leq \mathbb{E}\left[\mathbb{E}\left[Z_{n} \mid \Pi\right]^{\vartheta} \mid Z_{0}=z, L_{n} \geq 0\right] \\
& =\mathbb{E}\left[z^{\vartheta} \exp \left(\vartheta S_{n}\right) \mid L_{n} \geq 0\right] \leq z \mathbb{E}\left[\exp \left(\vartheta S_{n}\right) \mid L_{n} \geq 0\right]
\end{aligned}
$$

In view of Corollary 3.3 there is a $c^{\prime}>0$ such that $\eta_{n}(z) \leq c^{\prime} z$ for $\vartheta<\beta$ and $n \geq 1$, therefore

$$
\mathbb{E}\left[\eta_{n-k}\left(Z_{k}\right) ; \tau_{k}=k\right] \leq c^{\prime} \mathbb{E}\left[Z_{k} ; \tau_{k}=k\right]=c^{\prime} \mathbb{E}\left[e^{S_{k}} ; \tau_{k}=k\right] \leq c^{\prime \prime} \mathbb{P}\left\{L_{k} \geq 0\right\}
$$

As in the proof of the last lemma this implies

$$
\mathbb{E}\left[Z_{n}^{\vartheta}\right] \leq c \mathbb{P}\left\{L_{n} \geq 0\right\}
$$

for a suitable $c>0$. In view of Theorem 1.1 it follows that $\mathbb{E}\left[Z_{n}^{\vartheta} \mid Z_{n}>0\right]$ is bounded for $\vartheta<\beta$.

This also gives tightness of the distributions of $Z_{n}$, given $Z_{n}>0$. From the last lemma we see that $\mathbb{P}\left\{Z_{n}=a \mid Z_{n}>0\right\}$ is convergent for $a \geq 1$. This completes the proof.

Proof of Theorem 1.4 First we consider

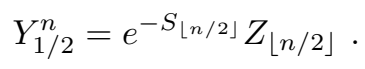

We show that

$$
\mathcal{L}\left(Y_{1 / 2}^{n} \mid Z_{n}>0\right) \stackrel{d}{\rightarrow} W,
$$

where $W$ is an a.s. positive random variable. 
Let $\chi: \mathbb{R} \rightarrow \mathbb{R}$ be bounded and continuous. As above in Lemma 3.6 we consider the decomposition

$$
\mathbb{E}\left[\chi\left(Y_{1 / 2}^{n}\right) ; Z_{n}>0\right]=\sum_{k=0}^{n} \mathbb{E}\left[\chi\left(Y_{1 / 2}^{n}\right) ; Z_{n}>0, \tau_{k}=k, \min _{k<l \leq n} S_{l} \geq S_{k}\right] .
$$

Again we devide it into three parts

$$
\mathbb{E}\left[\chi\left(Y_{1 / 2}^{n}\right) ; Z_{n}>0\right]=\sum_{k=0}^{m-1} \ldots+\sum_{k=m}^{n-m} \ldots+\sum_{j=0}^{m-1} \ldots
$$

with $m<\lfloor n / 2\rfloor$ fixed.

For the terms in the first sum $\sum_{k=0}^{m-1} \ldots$ we use the formula

$$
\begin{aligned}
\mathbb{E}\left[\chi\left(Y_{1 / 2}^{n}\right) ; Z_{n}>0, \tau_{k}\right. & \left.=k, \min _{k<l \leq n} S_{l} \geq S_{k}\right] \\
& =\mathbb{E}\left[\xi_{n, k}\left(Z_{k}, S_{k}\right) ; \tau_{k}=k\right] \mathbb{P}\left\{L_{n-k} \geq 0\right]
\end{aligned}
$$

with

$$
\xi_{n, k}(z, r)=\mathbb{E}\left[\chi\left(e^{-r} e^{-S_{m_{n-k}}} Z_{m_{n-k}}\right) ; Z_{n-k}>0 \mid L_{n-k} \geq 0, Z_{0}=z\right] .
$$

and $m_{n}=\lfloor(n+k) / 2\rfloor-k$. From Lemma 3.4 we see that this expression is convergent for $n \rightarrow \infty$, thus $\sum_{k=0}^{m-1} \ldots$ can be treated just as in the proof of Lemma 3.6 .

For the terms in the second sum $\sum_{k=m}^{n-m} \ldots$ we use the estimate

$$
\begin{aligned}
\mid \mathbb{E}\left[\chi\left(Y_{1 / 2}^{n}\right) ; Z_{n}>0,\right. & \left.\tau_{k}=k, \min _{k<l \leq n} S_{l} \geq S_{k}\right] \mid \\
& \leq \sup |\chi| \mathbb{P}\left\{Z_{n}>0, \tau_{k}=k, \min _{k<l \leq n} S_{l} \geq S_{k}\right\} .
\end{aligned}
$$

As in Lemma 3.6 we may conclude that the second sum becomes negligible by choosing $m$ sufficiently large.

For the terms of the third sum $\sum_{j=0}^{m-1} \ldots$ we use the formula

$$
\begin{aligned}
\mathbb{E}\left[\chi\left(Y_{1 / 2}^{n}\right) ; Z_{n}>0, \tau_{n-j}=n-j,\right. & \left.\min _{n-j<l \leq n} S_{l} \geq S_{n-j}\right] \\
& =\mathbb{E}\left[\chi\left(Y_{1 / 2}^{n}\right) \xi_{j}\left(Z_{n-j}\right) ; \tau_{n-j}=n-j\right]
\end{aligned}
$$

with

$$
\xi_{j}(z)=\mathbb{P}\left\{Z_{j}>0, L_{j} \geq 0 \mid Z_{0}=z\right\} .
$$

Now we may apply Lemma 3.5. Altogether (3.8) is proven. Also the statement $W>0$ a.s. follows from Lemma 3.4 and 3.5 
It remains to show that for $\varepsilon>0$

$$
\lim _{n \rightarrow \infty} \mathbb{P}\left\{\sup _{0 \leq t \leq 1}\left|Y_{t}^{n}-Y_{0}^{n}\right|>\varepsilon \mid Z_{n}>0\right\}=0 .
$$

First we consider a fixed environment. Then $\left(e^{-S_{i}} Z_{i}\right)$ is a martingale, thus applying the Doob inequality we obtain for $k<r_{n}$

$$
\begin{aligned}
\mathbb{P}\left\{\sup _{0 \leq t \leq 1}\left|Y_{t}^{n}-Y_{0}^{n}\right|>\right. & \left.\varepsilon \mid Z_{k}=z, \Pi\right\} \\
& \leq \varepsilon^{-2} \mathbb{E}\left[\left(e^{-S_{n-r_{n}}} Z_{n-r_{n}}-e^{-S_{r_{n}}} Z_{r_{n}}\right)^{2} \mid Z_{k}=z, \Pi\right] .
\end{aligned}
$$

Also a straightforward calculation gives for $i \geq k$

$$
\begin{aligned}
\mathbb{E}\left[\left(e^{-S_{i+1}} Z_{i+1}-\right.\right. & \left.\left.e^{-S_{i}} Z_{i}\right)^{2} \mid Z_{k}=z, \Pi\right] \\
& =z e^{-S_{k}}\left(\eta_{i+1} e^{-S_{i}}+e^{-S_{i+1}}-e^{-S_{i}}\right) .
\end{aligned}
$$

Given $\Pi$ and $Z_{k}=z$ the process $\left(e^{-S_{i}} Z_{i}\right)$ is therefore a $L_{2}$-martingale. Consequently

$$
\begin{aligned}
\mathbb{E}\left[\left(e^{-S_{n-r_{n}}} Z_{n-r_{n}}\right.\right. & \left.\left.-e^{-S_{r_{n}}} Z_{r_{n}}\right)^{2} \mid Z_{k}=z, \Pi\right] \\
& =\sum_{i=r_{n}}^{n-r_{n}-1} \mathbb{E}\left[\left(e^{-S_{i+1}} Z_{i+1}-e^{-S_{i}} Z_{i}\right)^{2} \mid Z_{k}=z, \Pi\right] \\
& \leq z e^{-S_{k}}\left(\sum_{i=r_{n}}^{n-r_{n}-1} \eta_{i+1} e^{-S_{i}}+e^{-S_{n-r_{n}}}\right) .
\end{aligned}
$$

Letting

$$
\begin{aligned}
& U_{n}:=\sum_{i=r_{n}}^{\lfloor n / 2\rfloor} \eta_{i+1} e^{-S_{i}}, V_{n}:=\sum_{i=r_{n}+1}^{\lceil n / 2\rceil} \eta_{i} e^{S_{i}}+e^{S_{r_{n}}} \\
& \varphi(u, v, x):=\varepsilon^{-2}\left(u+v e^{-x}\right)^{+}
\end{aligned}
$$

we obtain altogether (recall the definition of $\tilde{V}_{n}$ from the proof of Lemma 3.4)

$$
\mathbb{P}\left\{\sup _{0 \leq t \leq 1}\left|Y_{t}^{n}-Y_{0}^{n}\right|>\varepsilon \mid Z_{k}=z, \Pi\right\} \leq\left(z e^{-S_{k}} \varphi\left(U_{n}, \tilde{V}_{n}, S_{n}\right)\right) \wedge 1 .
$$


Once again we proceed in the by now established manner:

$$
\begin{aligned}
\mathbb{P}\left\{\sup _{0 \leq t \leq 1}\left|Y_{t}^{n}-Y_{0}^{n}\right|>\varepsilon, Z_{n}>0\right\} \\
=\sum_{k=0}^{n} \mathbb{P}\left\{\sup _{0 \leq t \leq 1}\left|Y_{t}^{n}-Y_{0}^{n}\right|>\varepsilon, Z_{n}>0, \tau_{k}=k, \min _{k<l \leq n} S_{l} \geq S_{k}\right\} \\
\leq \sum_{k=0}^{m-1} \mathbb{P}\left\{\sup _{0 \leq t \leq 1}\left|Y_{t}^{n}-Y_{0}^{n}\right|>\varepsilon, \tau_{k}=k, \min _{k<l \leq n} S_{l} \geq S_{k}\right\} \\
\quad+\sum_{k=m}^{n-m} \mathbb{P}\left\{Z_{n}>0, \tau_{k}=k, \min _{k<l \leq n} S_{l} \geq S_{k}\right\} \\
\quad+\sum_{j=0}^{m-1} \mathbb{P}\left\{\sup _{0 \leq t \leq 1}\left|Y_{t}^{n}-Y_{0}^{n}\right|>\varepsilon, Z_{n-j}>0, \tau_{n-j}=n-j\right\}
\end{aligned}
$$

As to the $\operatorname{sum} \sum_{k=0}^{m-1} \cdots$

$$
\begin{aligned}
\mathbb{P}\left\{\sup _{0 \leq t \leq 1}\left|Y_{t}^{n}-Y_{0}^{n}\right|>\varepsilon, \tau_{k}=k, \min _{k<l \leq n} S_{l} \geq S_{k}\right\} \\
\quad \leq \mathbb{E}\left[\mathbb{P}\left\{\sup _{0 \leq t \leq 1}\left|Y_{t}^{n}-Y_{0}^{n}\right|>\varepsilon \mid Z_{k}, \Pi\right\} ; \min _{k<l \leq n} S_{l} \geq S_{k}\right] \\
\leq \mathbb{E}\left[\left(Z_{k} e^{-S_{k}} \varphi\left(U_{n}, \tilde{V}_{n}, S_{n}\right)\right) \wedge 1 ; \min _{k<l \leq n} S_{l} \geq S_{k}\right] .
\end{aligned}
$$

Next $\mathbb{E}\left[\left(Z_{k} e^{-S_{k}} \varphi\left(U_{n}, \tilde{V}_{n}, S_{n}\right)\right) \wedge 1 \mid \Pi\right] \leq \varphi\left(U_{n}, \tilde{V}_{n}, S_{n}\right) \wedge 1$ because of Jensen's inequality applied to the concave function $x \mapsto x \wedge 1$. Thus we obtain

$$
\begin{aligned}
\mathbb{P}\left\{\sup _{0 \leq t \leq 1} \mid Y_{t}^{n}\right. & \left.-Y_{0}^{n} \mid>\varepsilon, \tau_{k}=k, \min _{k<l \leq n} S_{l} \geq S_{k}\right\} \\
& \leq \mathbb{E}\left[\varphi\left(U_{n}, \tilde{V}_{n}, S_{n}\right) \wedge 1 ; \min _{k<l \leq n} S_{l} \geq S_{k}\right] \\
& =\mathbb{E}\left[\left(e^{-S^{\prime}} \varphi\left(U_{n-k}, \tilde{V}_{n-k}, S_{n-k}\right)\right) \wedge 1 ; L_{n-k} \geq 0\right]
\end{aligned}
$$

where in the last expectation $S^{\prime}$ is distributed as $S_{k}$ before and independent from the other terms and also $r_{n-k}$ is replaced by $r_{n}-k$. Now $S_{n} \rightarrow-\infty$ $\mathbf{P}^{-}$-a.s. (compare Lemma 2.6 in [4]). This together with Lemma 3.1 gives that $U_{n} \rightarrow 0 \mathbf{P}^{+}$-a.s. and $V_{n} \rightarrow 0 \mathbf{P}_{x}^{-}$-a.s. From Theorem 2.7 it follows that

$$
\mathbb{P}\left\{\sup _{0 \leq t \leq 1}\left|Y_{t}^{n}-Y_{0}^{n}\right|>\varepsilon, \tau_{k}=k, \min _{k<l \leq n} S_{l} \geq S_{k}\right\}=o\left(\mathbb{P}\left\{L_{n} \geq 0\right\}\right) .
$$

Thus the sum $\sum_{k=0}^{m-1} \ldots$ is negligible. As we already know, the same is true for $\sum_{k=m}^{n-m} \ldots$ by choosing $m$ large. As to $\sum_{j=0}^{m-1} \ldots$ we obtain by means of Hölder's 
inequality for conjugate numbers $p, q>1$

$$
\begin{aligned}
\mathbb{P}\left\{\sup _{0 \leq t \leq 1}\left|Y_{t}^{n}-Y_{0}^{n}\right|>\varepsilon, Z_{n}>0 \mid \Pi\right\} \\
\quad \leq \mathbb{E}\left[Z_{n}^{1 / p} ; \sup _{0 \leq t \leq 1}\left|Y_{t}^{n}-Y_{0}^{n}\right|>\varepsilon \mid \Pi\right] \\
\leq \mathbb{E}\left[Z_{n} \mid \Pi\right]^{1 / p} \mathbb{P}\left\{\sup _{0 \leq t \leq 1}\left|Y_{t}^{n}-Y_{0}^{n}\right|>\varepsilon \mid \Pi\right\}^{1 / q} \\
\quad \leq \exp \left(S_{n} / p\right)\left(\varphi\left(U_{n}, \tilde{V}_{n}, S_{n}\right) \wedge 1\right)^{1 / q}
\end{aligned}
$$

and consequently

$$
\begin{aligned}
\mathbb{P}\left\{\sup _{0 \leq t \leq 1}\left|Y_{t}^{n}-Y_{0}^{n}\right|>\varepsilon, Z_{n-j}>0, \tau_{n-j}=n-j\right\} \\
\quad \leq \mathbb{E}\left[\exp \left(S_{n-j} / p\right)\left(\varphi\left(U_{n-j}, \tilde{V}_{n-j}, S_{n-j}\right) \wedge 1\right)^{1 / q} ; \tau_{n-j}=n-j\right],
\end{aligned}
$$

where $r_{n-j}$ is again replaced by $r_{n}-j$. Now we choose $p$ such that $1 / p>\beta$. Then, switching once more to the tilted measure $\mathbf{E}$, we may apply Theorem 2.8 again to obtain

$$
\mathbb{P}\left\{\sup _{0 \leq t \leq 1}\left|Y_{t}^{n}-Y_{0}^{n}\right|>\varepsilon, Z_{n-j}>0, \tau_{n-j}=n-j\right\}=o\left(\mathbb{P}\left\{L_{n} \geq 0\right\}\right) .
$$

Altogether

$$
\mathbb{P}\left\{\sup _{0 \leq t \leq 1}\left|Y_{t}^{n}-Y_{0}^{n}\right|>\varepsilon \mid Z_{n}>0\right\}=o\left(\mathbb{P}\left\{L_{n} \geq 0\right\}\right)=o(1)
$$

and this gives (3.9).

\section{References}

[1] Afanasyev, V. I. (1980). Limit theorems for a conditional random walk and some applications. Diss. Cand. Sci., Moscow, MSU.

[2] Afanasyev, V. I. (1990). On a maximum of a transient random walk in random environment. Theory Probab. Appl. 35, 205-215.

[3] Afanasyev, V. I. (1998). Limit theorems for a moderately subcritical branching process in a random environment. Discrete Math. Appl. 8, 55-52.

[4] Afanasyev, V. I., Geiger, J., Kersting, G. and Vatutin, V. A. (2005). Criticality for branching processes in random environment. Ann. Probab. 33, 645-673. 
[5] Afanasyev, V. I., Geiger, J., Kersting, G. and Vatutin, V. A. (2005). Functional limit theorems for strongly subcritical branching processes in random environment. Stoch. Proc. Appl. 115, 1658-1676

[6] Agresti, A. (1975). On the extinction times of varying and random environment branching processes. J. Appl. Probab. 12, 39-46.

[7] Athreya, K.B. and Karlin, S. (1971). On branching processes with random environments: I, II, Ann. Math. Stat. 42, 1499-1520, 1843-1858.

[8] Athreya, K. B. And Ney, P. (1972). Branching processes. Springer, Berlin.

[9] Bertoin, J., Doney, R.A. (1994) On conditioning a random walk to stay non-negative. Ann. Probab. 22, $2152-2167$.

[10] Bingham, N.H., Goldie, C.M. And Teugels, J.L. (1987) Regular variation. Cambridge University Press, Cambridge.

[11] Birkner, M., Geiger, J. And Kersting, G. (2005). Branching processes in random environment - a view on critical and subcritical cases. Proceedings of the DFG-Schwerpunktprogramm Interacting Stochastic Systems of High Complexity, Springer, Berlin, 265-291.

[12] Böinghoff, C. And Kersting, G. (2009). On large deviations of branching processes in a random environment - offspring distributions having at most geometric tails. Stoch. Proc. Appl. 120, 2064-2077.

[13] Chover, J., Ney, P. And Wainger, S. (1973) Functions of Probability measures. J. Analyse Math. 26, 255-302.

[14] Dekking, F.M. (1988). On the survival probability of a branching process in a finite state i.i.d. environment. Stoch. Proc. Appl. 27, 151-157.

[15] Doney, R.A. (1977). A note on a condition satisfied by certain random walks. J. Appl. Probab. 14, 843-849.

[16] Doney, R.A. (2010). Local behaviour of first passage probabilities. preprint, arXiv:1006.5316v1 
[17] Dyakonova, E.E., Geiger, J. and Vatutin, V.A. (2004). On the survival probability and a functional limit theorem for branching processes in a random environment. Markov Process. Relat. Fields, 10, 289-306.

[18] Feller, W. (1971) An Introduction to Probability Theory and Its Applications, Volume II. John Wiley and Sons, New York.

[19] Geiger, J., Kersting, G. and Vatutin, V.A. (2003). Limit theorems for subcritical branching processes in random environment. Ann. I.H. Poincaré (B). 39, 593-620.

[20] Hirano, K. (1998). Determination of the Limiting Coefficient for Exponential Functionals of Random Walks with Positive Drift. J. Math. Sci. Univ. Tokyo 5, 299-332.

[21] Iglehart, D. L. (1974). Random walks with negative drift conditioned to stay positive. J. Appl. Probab. 11, 742-751.

[22] Keener, R. W. (1992). Limit theorems for random walks conditioned to stay positive. Ann. Probab. 20, 801-824.

[23] Smith, W.L. And Wilkinson, W.E. (1969) On branching processes in random environments. Ann. Math. Stat. 40, 814-827.

Department of Discrete Mathematics

Steklov Institute

8 Gubkin Street, 117966 Moscow, GSP-1, Russia

viafan@mail.ru,vatutin@mi.ras.ru

Fachbereich Mathematik

Universität Frankfurt

Fach 187, D-60054 Frankfurt am Main, Germany

kersting@math.uni-frankfurt.de, boeinghoff@math.uni-frankfurt.de 